\title{
Local incompressibility estimates for the Laughlin phase
}

\author{
Elliott H. Lieb ${ }^{1}$, Nicolas Rougerie ${ }^{2}$, Jakob Yngvason ${ }^{3,4}$ \\ ${ }^{1}$ Departments of Mathematics and Physics, Princeton University, Princeton, NJ 08544, USA. \\ E-mail: lieb@princeton.edu \\ 2 LPMMC (UMR 5493), Université Grenoble 1 \& CNRS, B.P. 166, 38042 Grenoble, France. \\ E-mail: nicolas.rougerie@grenoble.cnrs.fr \\ 3 Fakultät für Physik, Universität Wien, Boltzmanngasse 5, 1090 Vienna, Austria \\ 4 Erwin Schrödinger Institute for Mathematical Physics, Boltzmanngasse 9, 1090 Vienna, Austria. \\ E-mail: jakob.yngvason@univie.ac.at
}

\begin{abstract}
We prove sharp density upper bounds on optimal length-scales for the ground states of classical 2D Coulomb systems and generalizations thereof. Our method is new, based on an auxiliary Thomas-Fermi-like variational model. Moreover, we deduce density upper bounds for the related low-temperature Gibbs states. Our motivation comes from fractional quantum Hall physics, more precisely, the perturbation of the Laughlin state by external potentials or impurities. These give rise to a class of many-body wavefunctions that have the form of a product of the Laughlin state and an analytic function of many variables. This class is related via Laughlin's plasma analogy to Gibbs states of the generalized classical Coulomb systems we consider. Our main result shows that the perturbation of the Laughlin state cannot increase the particle density anywhere, with implications for the response of FQHE systems to external perturbations.
\end{abstract}

\section{Contents}

1. Introduction . . . . . . . . . . . . . . . . . . . . 432

2. Main Results . . . . . . . . . . . . . . . . . . . . . . . 435

2.1 Universal density bounds . . . . . . . . . . . . . . . . . 435

2.2 Potential energy estimates . . . . . . . . . . . . . . . . . . 436

2.3 Proof outline: screening properties in the plasma analogy . . . . . . . 438

3. Incompressible 2D Thomas-Fermi Molecules . . . . . . . . . . . . . . . 441

3.1 Existence and uniqueness of a minimizer . . . . . . . . . . . . . . . . . 443

3.2 The Thomas-Fermi potential . . . . . . . . . . . . . . . . 446

4. Local Density Upper Bounds for Classical Ground States . . . . . . . . . 450

4.1 Exclusion by screening . . . . . . . . . . . . . . . . . . 450

4.2 Exclusion rule and density bounds . . . . . . . . . . . . . . . . . . 452

4.3 Applications ......................... . . . 456

5. Local Density Bounds for Classical Gibbs States . . . . . . . . . . . . . . . . . . 457

5.1 Conditional local density bound . . . . . . . . . . . . . . . 457 
5.2 Localization procedure . . . . . . . . . . . . . . . . . 461

6. Energy in Confining Potentials . . . . . . . . . . . . . . 465

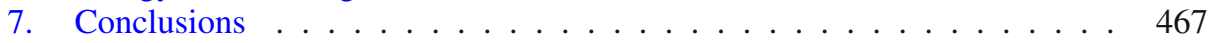

References . . . . . . . . . . . . . . . . . . . . . . 468

\section{Introduction}

The fractional quantum Hall effect (FQHE) $[25,29,33,69]$ is a remarkable feature of the transport properties of $2 \mathrm{D}$ electron gases under strong perpendicular magnetic fields and at low temperatures. Soon after its experimental discovery [71], it was recognized in the seminal works of Laughlin [31,32] that the origin of the effect lies in the emergence of a new, strongly correlated, phase of matter. The latter has been argued to host elementary excitations with fractional charge, a fact that was later experimentally confirmed [21,47,63]. Even more fascinating, but still lacking an experimental confirmation, is the possibility that these excitations (quasi-particles) are anyons $[6,45,73]$, i.e. have quantum statistics different from those of bosons and fermions, the only known types of fundamental particles. Due to these exciting prospects, it is an ongoing quest in condensed matter physics to generalize FQHE physics to other, more flexible, contexts than the 2D electron gas $[9,11,20,49,72]$.

One of the distinctive features of strongly correlated $\mathrm{FQH}$ states is that they are incompressible liquids, rendering them very robust against perturbations and external fields. Incompressibility, in the form relevant to the FQHE, has in fact two aspects:

1. The proposed strongly correlated $\mathrm{FQHE}$ wave functions are approximate ground states for the many-body Hamiltonian of the system at hand. Their energy is separated from the rest of the spectrum by a gap independent of volume and particle number.

2. Modifications of model $\mathrm{FQHE}$ ground states that stay within the (highly degenerate) ground eigenspace of the many-body Hamiltonian cannot increase the local oneparticle density beyond a fixed value.

These two aspects are very non-trivial to check, particularly since we are talking about strongly correlated states of matter for which mean-field descriptions in terms of independent particles are not adequate. The main evidence so far in favor of Properties 1 and $\mathbf{2}$ has been experimental and numerical and it is an important theoretical challenge to improve on this.

The main paradigms of the FQHE are encoded in Laughlin's wave-function, the simplest FQH state:

$$
\Psi_{\text {Lau }}\left(z_{1}, \ldots, z_{N}\right)=c_{\text {Lau }} \prod_{i<j}\left(z_{i}-z_{j}\right)^{\ell} e^{-B \sum_{i=1}^{N}\left|z_{i}\right|^{2} / 4} .
$$

Here $z_{1}, \ldots, z_{N} \in \mathbb{C}$ are the positions of $N$ particles moving in $\mathbb{R}^{2}$, identified with the complex plane, and the constant $c_{\text {Lau }}$ is a normalization factor (setting the $L^{2}$-norm equal to 1). For fermions, $\ell$ is odd and $\geq 3$ (the case $\ell=1$ corresponds to noninteracting fermions), while for bosons $\ell \geq 2$ is even. The function (1.1) has originally been proposed as a variational ansatz for the ground state of the many-body magnetic Schrödinger Hamiltonian ${ }^{1}$

\footnotetext{
${ }^{1}$ We use the label QM to distinguish the quantum mechanical Hamiltonian (1.2) from the classical Hamiltonians $H_{N}$ that will be introduced later in Sect. 2.3.
} 


$$
H_{N}^{\mathrm{QM}}=\sum_{j=1}^{N}\left[\left(-i \nabla_{j}-\frac{B}{2} x_{j}^{\perp}\right)^{2}+V\left(x_{j}\right)\right]+\sum_{1 \leq i<j \leq N} w\left(x_{i}-x_{j}\right)
$$

acting on $L^{2}\left(\mathbb{R}^{2 N}\right)$, the Hilbert space for $N$ 2D particles. Here $x^{\perp}$ denotes the vector $x \in \mathbb{R}^{2}$ rotated by $\pi / 2$ counter-clockwise, so that

$$
\operatorname{curl} \frac{B}{2} x^{\perp}=B
$$

and thus $\frac{B}{2} x^{\perp}$ is the vector potential of a uniform magnetic field, expressed in symmetric gauge.

To arrive at an ansatz of the form (1.1) one assumes that the energy scales are set, in order of importance, by

- the strength $B$ of a constant applied magnetic field perpendicular to the plane,

- the repulsive pair-interaction potential $w$,

- the external scalar potential $V$, representing trapping and/or disorder.

In the sequel we choose units so that the strength of the magnetic field is 2 and the magnetic length therefore $1 / \sqrt{2}$.

Since the magnetic field is the main player, the first reduction is to replace the state space $L^{2}\left(\mathbb{R}^{2 N}\right) \simeq \bigotimes^{N} L^{2}\left(\mathbb{R}^{2}\right)$ by $\bigotimes^{N} \mathfrak{H}$ where $(z$ is the complex coordinate in the plane)

$$
\mathfrak{H}=\left\{\psi(z)=f(z) e^{-|z|^{2} / 2} \in L^{2}\left(\mathbb{R}^{2}\right) \mid f \text { analytic }\right\}
$$

is the lowest Landau level (LLL), ground eigenspace of the magnetic Laplacian $\left(-i \nabla-\frac{B}{2} x^{\perp}\right)^{2}$. In other words, one reduces available one-body orbitals to those minimizing the magnetic kinetic energy. Spins are all aligned with the external magnetic field so that one only needs consider symmetric/antisymmetric wave-functions of $\bigotimes^{N} \mathfrak{H}$ to describe bosons/fermions.

Secondly, in order to suppress repulsive interactions, the many-body wave-function of the system should vanish when two particles meet. Combined with the regularity of the LLL orbitals, this forces the inclusion of Jastrow factors $\left(z_{i}-z_{j}\right)^{\ell}, \ell \in \mathbb{N}$ in the wave-function, leading to (1.1). The integer $\ell$ is chosen to accommodate symmetry (the wave-function must be symmetric/antisymmetric under coordinates exchanges $z_{i} \leftrightarrow z_{j}$ to describe bosons/fermions) and to fix the filling factor (number of particles per magnetic flux quantum) equal to $1 / \ell$. In the absence of an external potential, there is then no free variational parameter in the ansatz (1.1).

The proposed form (1.1) turns out to be sufficiently robust for serving as the basic ingredient of approximate ground states for large classes of repulsive interaction potentials $w$ and external potentials $V$.

A possible mathematical formulation of Property $\mathbf{1}$ is to consider a model pairpotential $w$ whose projection to the $N$-body LLL has (1.1) as an exact zero-energy ground-state $[26,48,70]$. The claim would then be that the next energy level is bounded below, independently of $N$. This problem has remained open so far, see $[39,58]$ for further discussion.

Property $\mathbf{2}$ is the main subject of the present contribution. To formulate it properly, we note that the arguments leading to choosing (1.1) as a variational ansatz leave the possibility to choose any $L^{2}$-normalized function of the form

$$
\Psi_{F}\left(z_{1}, \ldots, z_{N}\right)=F\left(z_{1}, \ldots, z_{N}\right) \Psi_{\text {Lau }}\left(z_{1}, \ldots, z_{N}\right)
$$


with $F$ analytic and symmetric under exchange of the $z_{i}$. This form exhausts the class of functions that minimize the magnetic kinetic energy and at the same time avoid repulsive interactions by vanishing at least as $\left(z_{i}-z_{j}\right)^{\ell}$ as $z_{i}$ and $z_{j}$ come together. In the bosonic case and with $\ell=2$ these are exactly the ground states of the contact interaction [58, Section 2.1]. The factor $F$ allows an adaption to an external potential. We shall refer to the class of states of the form (1.4) as fully correlated states.

Consider now the one-particle density

$$
\rho_{F}(z)=N \int_{\mathbb{R}^{2(N-1)}}\left|\Psi_{F}\left(z, z_{2}, \ldots, z_{N}\right)\right|^{2} d z_{2} \ldots d z_{N}
$$

of a state of the form (1.4). For $F=1$, i.e., for the Laughlin state (1.1), it was argued in [31] and proved rigorously in $[57,58]$ that the density takes essentially the constant value $1 /(\pi \ell)$ in the disk $D(0, \sqrt{\pi \ell N})$, and drops quickly to 0 outside of the disk. ${ }^{2}$ Property 2 can then roughly be formulated as

$$
\rho_{F} \lesssim \frac{1}{\pi \ell} \text { for any state of the form (1.4). }
$$

Some care has to be taken in formulating this rigorously for, in view of existing numerical simulations $[18,19]$, the symbol $\lesssim$ above cannot actually stand for a microscopic pointwise bound for finite $N$.

In this paper we improve and generalize results of [59,60] (see also [55, Chapter 3] for further discussion) by proving that (1.6) holds in the sense of local averages over length scales $\gg N^{1 / 4}$, which are much smaller than the extension of the Laughlin state itself, of order $N^{1 / 2}$. In view of recent results [7,34], which can be applied to the pure Laughlin state, i.e., $F=1$, it is natural to conjecture that (1.6) in fact holds on any length scale $N^{\alpha}, \alpha>0$. In order to understand the response of the Laughlin state to external potentials, it is essential, however, to know that the density bound holds for all analytic factors $F$. The methods of $[7,34]$, in contrast to those we develop here, do not apply in such generality.

The bound (1.6) is an expression of the robustness of the Laughlin state against perturbations by external fields because it forbids a fully-correlated state to accommodate variations of an external potential by concentrating arbitrarily large portions of its mass in energetically favorables places: Any redistribution of the mass must respect the same density bound (1.6) as the Laughlin state.

On the other hand, it has recently been proved in [61] that, if (1.6) is known to hold for all strongly correlated states, then the minimal energy in an external potential within this class of states can asymptotically be attained in states saturating the bound (1.6) and having the simple form

$$
\Psi_{f}\left(z_{1}, \ldots, z_{N}\right)=\prod_{j=1}^{N} f\left(z_{j}\right) \Psi_{\mathrm{Lau}}\left(z_{1}, \ldots, z_{N}\right)
$$

where $f$ is a polynomial of a single variable. Physically, this means that it is never favorable to add more correlations to the Laughlin state in order to accommodate an external potential, provided the latter is weak enough so that it does not make the ground state jump across the energy gap assumed in Property 1.

\footnotetext{
2 This is a consequence of concentration of measure results for $\beta$-ensembles, see [66,67] for review and references.
} 
The zeros $a_{j} \in \mathbb{C}$ of the polynomial $f$,

$$
f(z)=\text { const. } \prod_{j=1}^{J}\left(z-a_{j}\right),
$$

are interpreted as the locations of quasi-holes, each carrying a charge $1 / \ell$.

If the locations $a_{1}, \ldots, a_{J}$ are treated quantum-mechanically instead of classically as above, one should expect that the quasi-holes behave as anyons $[6,45,73]$ with statistics parameter $-1 / \ell$.

The results of the present paper, together with [61], confirm rigorously Laughlin's original intuition $[31,32]$ that the response of the Laughlin state to a disorder potential is to generate uncorrelated quasi-holes, which is also in accord with experimental studies [47].

In the next section we state our main results precisely and discuss them further (they were announced in the short paper [42]). The rest of the paper is devoted to their proofs.

\section{Main Results}

2.1. Universal density bounds. As discussed in the introduction (see also $[59,60]$ ), we assume that the magnetic field and repulsive interactions are strong enough, so that the ground state of the system has the form (1.4). We aim at proving that there is a universal upper bound to the local density of all such states, on scales much smaller than the total size of the system. Thus, let

$$
\Psi_{\text {Lau }}\left(z_{1}, \ldots, z_{N}\right)=c_{\text {Lau }} \prod_{i<j}\left(z_{i}-z_{j}\right)^{\ell} e^{-\sum_{i=1}^{N}\left|z_{i}\right|^{2} / 2}
$$

be the $L^{2}$-normalized Laughlin function with exponent $\ell \in \mathbb{N}$, in units where the magnetic length is $1 / \sqrt{2}$. As previously explained we consider analytic perturbations thereof:

$$
\mathcal{L}_{\ell}^{N}=\left\{\Psi_{F}=F\left(z_{1}, \ldots, z_{N}\right) \Psi_{\text {Lau }} \mid F \text { analytic and symmetric, }\left\|\Psi_{F}\right\|_{L^{2}\left(\mathbb{R}^{2 N}\right)}=1\right\} .
$$

For a function $\Psi_{F} \in \mathcal{L}_{\ell}^{N}$ we denote by

$$
\rho_{F}(z)=N \int_{\mathbb{R}^{2(N-1)}}\left|\Psi_{F}\left(z, z_{2}, \ldots, z_{N}\right)\right|^{2} d z_{2} \ldots d z_{N}
$$

the associated one-particle density. Our main result is a universal upper bound on $\rho_{F}$, holding on mesoscopic length scales:

Theorem 2.1 (Rigidity bound for fully-correlated states). For any $\alpha>1 / 4$, any disk $D$ of radius $N^{\alpha}$ and any (sequence of) states $\Psi_{F} \in \mathcal{L}_{\ell}^{N}$ we have

$$
\int_{D} \rho_{F} \leq \frac{1}{\pi \ell}|D|(1+o(1))
$$

where $|D|$ is the area of the disk and o(1) tends to zero as $N \rightarrow \infty$. 
More generally, for any open set with Lipschitz boundary $\Omega$, denote by $\Omega_{r}$ the set obtained by dilating $\Omega$ around some origin by a factor $r=N^{\alpha}$. Then, for any $\alpha>1 / 4$

$$
\int_{\Omega_{r}} \rho_{F} \leq \frac{1}{\pi \ell}\left|\Omega_{r}\right|(1+o(1))
$$

as $N \rightarrow \infty$.

2.2. Potential energy estimates. With Theorem 2.1 in hand, we can return to the original physical problem (1.2) in the presence of an external potential $V$. If the magnetic kinetic energy is frozen by restricting one-body orbitals to the LLL, and if we further assume that the interaction energy is also frozen by restricting to (2.2), the only non-trivial energy term left in the Hamiltonian is set by $V$. An interesting problem consists in studying the minimization of this energy amongst fully-correlated states:

$$
E_{V}(N, \ell):=\inf \left\{\int_{\mathbb{R}^{2}} V(z) \rho_{F}(z) d z, \rho_{F} \text { as in }(2.3), \Psi_{F} \in \mathcal{L}_{\ell}^{N}\right\} .
$$

This infimum will not necessarily be attained, but it is easy to see that, for fixed $N$, the density $\rho_{F}$ is bounded in $L^{\infty}$ uniformly in $F$ (this follows from the fact that all onebody orbitals are in the LLL [14], see the discussion in [59, Remark 2.2]). Therefore the infimum exists for any $V$ such that its negative part, $V_{-}$, is in $L^{1}\left(\mathbb{R}^{2}\right)$.

In view of Theorem 2.1, it is fairly natural to expect a lower bound to (2.6) in terms of the "bathtub energy", defined as [41, Theorem 1.14]

$$
E_{V}^{\mathrm{bt}}(\ell):=\min \left\{\int_{\mathbb{R}^{2}} V \rho \mid 0 \leq \rho \leq \frac{1}{\pi \ell}, \int_{\mathbb{R}^{2}} \rho=N\right\} .
$$

We can prove this for a large class of external potentials $V$, that we now describe. Since the typical extension of the Laughlin state is of order $\sqrt{N}$, it is more convenient to state our assumptions in terms of a scaled version of the potential.

Assumption 2.2 (The external potential). Let $V \in C^{2}\left(\mathbb{R}^{2}\right)$ be a (sequence of) potential(s) that we write in the manner

$$
V(x)=U\left(\frac{x}{\sqrt{N}}\right)
$$

for a (possibly $N$-dependent) $U \in C^{2}\left(\mathbb{R}^{2}\right)$. Assume that there exists a fixed radius $R>0$ and an $\alpha>0$ such that

\section{(1) Local behavior:}

$$
\|\nabla U\|_{L^{\infty}(D(0, R))} \leq C N^{1 / 2-\alpha}, \quad\|\Delta U\|_{L^{\infty}(D(0, R))} \leq C N^{1-2 \alpha} .
$$

(2) Growth at infinity: $U \geq \underline{U}$ outside of $D(0, R)$ for a potential $\underline{U} \in C^{2}\left(\mathbb{R}^{2}\right)$ satisfying

$$
\|\nabla \underline{U}\|_{L^{\infty}\left(\mathbb{R}^{2}\right)} \leq C, \quad\|\Delta \underline{U}\|_{L^{\infty}\left(\mathbb{R}^{2}\right)} \leq C
$$

and

$$
\int_{\mathbb{R}^{2}} \exp (-\underline{U}) \leq C
$$

independently of $N$. 
A typical example we have in mind is a potential $V$ of the sort

$$
V(x)=V_{\text {trap }}(x)+V_{\text {disorder }}(x)=U_{\text {trap }}\left(\frac{x}{\sqrt{N}}\right)+U_{\text {disorder }}\left(\frac{x}{\sqrt{N}}\right)
$$

where the part $U_{\text {trap }}$ represents trapping in a given sample, and satisfies Item 2 of the assumptions, whereas $U_{\text {disorder }}$ represents some disorder due to impurities in the sample, is compactly supported in a fixed disk and satisfies Item 1 of the assumption.

Item 1 means that we consider a potential $V$ whose variations happen on length scales $O\left(N^{\alpha}\right), \alpha>0$, the best scale we may hope for (but cannot quite reach) in our density bounds, Theorem 2.1. Note that this should not be interpreted as saying that the potential energy bounds we are about to state work on the optimal length scale, see Remark 2.4 below. Item 2 is essentially a mild trapping assumption. It is clearly satisfied by any regular enough function $U$ growing at least like $c \log |x|, c>2$ at infinity.

We shall prove the following corollary, which improves [60, Theorem 1.1]:

Corollary 2.3 (Lower bound to the potential energy). Let $V$ be as in Assumption 2.2. Then

$$
E_{V}(N, \ell) \geq E_{V}^{\mathrm{bt}}(\ell)(1+o(1))+o(N)
$$

as $N \rightarrow \infty$.

Remark 2.4. (Affordable length scales) In accordance with the limitations of Theorem 2.1, the above result is meaningful when $\alpha>1 / 4$ in Assumption 2.2, i.e., the potential varies on length scales much larger than $N^{1 / 4}$. To appreciate this, recall [41, Theorem 1.14] that the minimizer of the bathtub energy takes only the values 0 and $(\pi \ell)^{-1}$ almost everywhere. Under Assumption 2.2, the typical length scale of the potential $V$ is $L=N^{\alpha}$. It can have a variation of order $r L$ in a disk of radius $r$. For comparison (and without loss of generality) let us set the reference energy to be the minimum of $V$ in the disk. The difference in bathtub energy according to whether the minimizer is, say, equal to 0 or $(\pi \ell)^{-1}$ everywhere in said disk cannot be larger than $O\left(r^{3} L\right)$ (area times maximal value of the potential). Pick now $r=L$. For the energy difference to dominate the error term $o(N)$ in (2.13), we need $L^{4} \gg N$, that is $N^{4 \alpha} \gg N$, and this is guaranteed only if $\alpha>1 / 4$. The bound (2.13) thus captures the behavior of the bathtub minimizer on its smallest length scale (i.e., that of $V$ ) only if the latter is $\gg N^{1 / 4}$. $\diamond$

In the recent paper [61], Theorem 2.3, an upper energy bound matching the lower bound (2.13) has been proved:

Proposition 2.5 (Upper bound to the potential energy, [61]). Under the additional ${ }^{3}$ condition that $U$ in Assumption 2.2 is fixed ( $N$-independent), we have

$$
E_{V}(N, \ell) \leq E_{V}^{\mathrm{bt}}(\ell)\left(1+O\left(N^{-1 / 4}\right)\right) .
$$

The above is obtained by using trial states of the form (1.7). For the special case where $V$ is radially symmetric and monotonously increasing in the radial variable, or is of a Mexican-hat form, such an upper bound was proved earlier in [59,60]. A remarkable consequence is that the Laughlin state stays an approximate ground state in any radial increasing potential, however steep. We refer to $[59,60]$ again and to the companion paper [42] for further discussion of the significance of this.

\footnotetext{
${ }^{3}$ Mostly technical, see [61] for details.
} 
2.3. Proof outline: screening properties in the plasma analogy. The backbone of the proof of Theorem 2.1 is Laughlin's plasma analogy, a very fruitful mapping of the many-body density of $\Psi_{\mathrm{Lau}}$, and more generally $\Psi_{F}$, to a problem in classical statistical mechanics, originating in [31,32]. As in our previous papers [57-60] we generalize this to any fully-correlated state (1.4). We rewrite the $N$-particle probability density of any fully correlated state $\Psi_{N}$ in the form of a Boltzmann-Gibbs factor

$$
\left|\Psi_{F}\left(z_{1}, \ldots, z_{N}\right)\right|^{2}=\frac{1}{\mathcal{Z}_{N}} \exp \left(-H_{N}\left(z_{1}, \ldots, z_{N}\right)\right)
$$

with a classical Hamiltonian

$$
H_{N}\left(z_{1}, \ldots, z_{N}\right)=\sum_{j=1}^{N}\left|z_{j}\right|^{2}-2 \ell \sum_{1 \leq i<j \leq N} \log \left|z_{i}-z_{j}\right|-2 \log \left|F\left(z_{1}, \ldots, z_{N}\right)\right| .
$$

To interpret (2.16) as an energy functional for $N$ classical positively charged particles with coordinates $z_{1}, \ldots, z_{N} \in \mathbb{C} \simeq \mathbb{R}^{2}$ we recall that Poisson's equation relates the electrostatic potential $\varphi$ generated by a charge distribution $\rho$ in the manner

$$
-\Delta \varphi=2 \pi \rho
$$

where $\Delta$ is the two-dimensional Laplacian. Thus one can see (2.16) as the energy of $N$ positively charged particles subject to

- the external potential generated by a constant background of opposite charge. Since

$$
-\Delta_{z_{j}}\left|z_{j}\right|^{2}=-4
$$

the potential $\left|z_{j}\right|^{2}$ can be interpreted as being generated by a uniform charge background of negative density $-2 / \pi$.

- pairwise Coulomb repulsion:

$$
-\Delta_{z_{j}}\left(-2 \ell \sum_{1 \leq i<j \leq N} \log \left|z_{i}-z_{j}\right|\right)=\sum_{i \neq j} 4 \pi \ell \delta_{z_{i}=z_{j}}
$$

thus the second term in (2.16) is the $2 \mathrm{D}$ Coulomb interaction between particles of identical charge $\sqrt{2 \ell}$.

- the potential generated by an essentially arbitrary, but positive, (many particle) charge distribution encoded in the (modulus of the) analytic function $F$. Indeed, for any $j=1 \ldots N$,

$$
-\Delta_{z_{j}}\left(-\log \left|F\left(z_{1}, \ldots, z_{N}\right)\right|\right) \geq 0 .
$$

This is, in fact, the only property of $F$ we use in our method. Equation (2.20) means that $-\log |F|$ is superharmonic in every variable, because $F$ is analytic. ${ }^{4}$

A plausibility argument for our main results goes as follows:

(1) The effective temperature in (2.15) is small compared to the total energy, so that the Boltzmann-Gibbs factor is essentially concentrated around minimizing configurations of the Hamiltonian (2.16).

\footnotetext{
4 One can write it in Weierstrass factorized form to see that (2.20) holds, or use the Cauchy integral formula for the value of an analytic function at the center of any disk and rely on the characterization of superharmonic functions in terms of averages in disks [41, Chapter 9].
} 
(2) For $F=1$, i.e. in the case of the pure Laughlin state, one should expect that the local density of points in a minimizing configuration is close to $1 /(\ell \pi)$. Indeed, this is the condition for the negatively charged fixed constant background (c.f. (2.18)) to efficiently screen the positively charged mobile particles $z_{j}, j=1 \ldots N$ (c.f. (2.19)).

(3) If $F \neq 1$ we add an additional positive charge distribution to the game because of (2.20). Since the mobile particles $z_{j}, j=1 \ldots N$ are positively charged in this representation, one can expect that such an addition will exert a repelling force on the density distribution of the mobile particles and reduce it, at least on the average.

This intuitive picture is, however, not as clear as it may look at first sight. If we imagine the potential term associated to $F$ as being generated by point-like positively charged particles, it is not obvious a priori why several such charges cannot work together and conspire to increase the density locally. Arranged tightly on a circle they could, perhaps, concentrate the density at a high value around the center of the circle. Note also that $-2 \log \left|F\left(z_{1}, \ldots, z_{N}\right)\right|$ is generated by a mobile charge distribution because $\Delta_{z_{j}} \log \left|F\left(z_{1}, \ldots, z_{N}\right)\right|$ is in general a function of $z_{1}, \ldots, z_{N}$ and only constant in special cases (essentially when $F=f^{\otimes N}$ is a tensor power, as in (1.7)). Thus, the positions of the additional charges are in general correlated with those of the actual charges. Moreover, as the example $F\left(z_{1}, \ldots, z_{N}\right)=\prod_{i} \exp \left(c z_{i}\right)$ shows, the area occupied by the bulk of the density can be stretched in one direction but compressed in another.

The heuristic argument (1)-(3) above is, in any case, certainly far from a rigorous proof of the bound (2.4). To obtain such a proof, the reduction to considering only ground states of (2.16) (rather than the full Gibbs state) presents, perhaps, the least difficulty. To appreciate this, it is helpful to rescale lengths: we can write

$$
\mu_{F}\left(z_{1}, \ldots, z_{N}\right):=N^{N}\left|\Psi_{F}\left(\sqrt{N} z_{1}, \ldots, \sqrt{N} z_{N}\right)\right|^{2}=\frac{1}{\tilde{\mathcal{Z}}_{N}} \exp \left(-N \tilde{H}_{N}\left(z_{1}, \ldots, z_{N}\right)\right)
$$

as a probability measure with

$$
\begin{aligned}
\tilde{H}_{N}\left(z_{1}, \ldots, z_{N}\right)= & \sum_{j=1}^{N}\left|z_{j}\right|^{2}-2 \frac{\ell}{N} \sum_{1 \leq i<j \leq N} \log \left|z_{i}-z_{j}\right| \\
& -\frac{2}{N} \log \left|F\left(\sqrt{N} z_{1}, \ldots, \sqrt{N} z_{N}\right)\right| .
\end{aligned}
$$

Thus, modulo a simple change of length-scales, we can reduce the aim to a statistical mechanics problem

- in mean-field scaling, the first and second sum in the above being of the same order for large $N$.

- with small effective temperature $T=N^{-1}$.

This makes it clear why mostly ground-state configurations are relevant for the proof, although some care is needed to estimate the effect of entropy terms in the classical free energy. In fact, the reduction to classical ground state configurations is responsible for the fact that our main result holds only on length scales $\gg N^{1 / 4}$. For ground-states instead of Gibbs states, the result holds on scales $\gg 1$. There is thus certainly room for improvement in this particular part of the proof, see Remark 2.6 below.

With the reduction to ground states of (2.16) out of the way, the main question is now to understand points (2) and (3) of the list above. In our approach, the solution is essentially based on screening properties of ground states of classical Coulomb systems, 
namely their tendency to achieve local neutrality in order for the total potential generated by the positively and negatively charged particles to compensate one another.

Consider a minimizing configuration $Z_{N}^{0}=\left\{z_{1}^{0}, \ldots, z_{N}^{0}\right\}$ for the classical Hamiltonian (2.16) (the labeling of the points is irrelevant by symmetry of (2.16) under permutations). To any subset $\left\{z_{1}^{0}, \ldots, z_{K}^{0}\right\}$ of the configuration (in fact, any $K$-tuple of points in $\mathbb{R}^{2}$ ) we associate an open screening region $\Sigma_{K}$ such that

$$
\Phi(x)=\int_{\Sigma_{K}} \frac{1}{\pi \ell} \log |x-y| d y-\sum_{j=1}^{K} \log \left|x-z_{k}\right| \begin{cases}=0 & \text { outside } \Sigma_{K} \\ >0 & \text { inside } \Sigma_{K}\end{cases}
$$

The above means that the total potential generated by the $K$ positive charges under consideration and a constant background of opposite charge density $(\pi \ell)^{-1}$ contained in $\Sigma_{K}$ vanishes identically outside $\Sigma_{K}$. The background density coming from the first term in (2.16) thus completely screens the $K$ point charges outside of the set $\Sigma_{K}$. Note that for (2.23) to hold we must have neutrality,

$$
\left|\Sigma_{K}\right|=\pi \ell K
$$

The sets $\Sigma_{K}$ are constructed in our approach via the minimization of an auxiliary functional bearing some formal resemblance to a $2 \mathrm{D}$ version of the usual Thomas-Fermi theory for molecules [40]. Accepting their existence, the rest of the proof has two main steps, going roughly as follows

- Given the screening region $\Sigma_{K}$ associated to a subset $\left\{z_{1}^{0}, \ldots, z_{K}^{0}\right\}$ of the minimizing configuration, no other point of a minimizing configuration of particles can lie within $\Sigma_{K}$. We refer to this as the exclusion property. It holds for ground states of (2.16), as we shall prove, because the third term herein is superharmonic.

- Any configuration of points satisfying the exclusion property must have its local density bounded above by $(\pi \ell)^{-1}$. If some region contains a larger density, consider a disk included in it. The screening region associated to the points contained in the disk would have, because of (2.24), to leak outside of the disk. It would then overlap with other points of the configuration, which is impossible because of the exclusion property.

The construction of the screening set, via a variant of Thomas-Fermi theory, is done in Sect. 3. Some care is needed in handling the auxiliary variational problem, because it is of a non-standard type. We next prove the local density bound for ground states of (2.16) in Sect. 4, following the above strategy. The application to low-temperature Gibbs states (2.15) occupies Sect. 5, where we prove Theorem 2.1. Finally we deduce Corollary 2.3 in Sect. 6.

Remark 2.6 (Recent related results). The classical Hamiltonian (2.16) and the associated Gibbs state (2.15) in the pure Laughlin case $F=1$ have attracted much attention in their own right. Apart from the connection with the FQHE we are primarily concerned about here, one can see them as basic models for trapped plasmas. In this context, a generalization often considered (2D log-gas or $2 \mathrm{D} \beta$-ensemble) is to replace $\left|z_{j}\right|^{2} \rightarrow V\left(z_{j}\right)$ in (2.22), with $V: \mathbb{R}^{2} \mapsto \mathbb{R}$ a general trapping potential. Minimizing configurations are then weighted Fekete sets [62], and Gibbs states for special values of the temperature are connected to certain random matrices ensembles [5,22].

For general $V$ the density is not flat: the limiting density of points is proportional to $\Delta V$ on its support. One expects the true minimizers and low-temperature Gibbs 
states to follow this profile on any microscopic length scale $\gg N^{-1 / 2}$ (in the scaled variables used in (2.22)). This has recently been proved in [4,53] for ground states (see also [50] for higher dimensional Coulomb and Riesz gases) and in [7,8,34,36] for Gibbs states. See also earlier partial results (not on the optimal scale) in $[51,56,65]$ and $[2,3,10,16,17,35,37,64]$ for related recent literature.

One can see our main result as a generalization of the fine-scale rigidity estimates of [4, $7,34,53]$ to the case of a non-trivial $F$. Motivated by FQHE physics, this generalization is our chief concern. For general analytic $F$ one should expect only local density upper bounds, but no corresponding lower bound in general. None of the available methods seem to apply here, but the new approach we sketched above might be of interest in the usual context with $F=1$ and a general $V$ in (2.22). Note that we obtain the expected density upper bound on essentially optimal length scales for minimizing configurations (see Proposition 4.6 below), but that it remains an open problem to do the same for Gibbs states, i.e. allow any $\alpha>0$ instead of only $\alpha>1 / 4$ in Theorem 2.1.

\section{Incompressible 2D Thomas-Fermi Molecules}

In this section we discuss a Thomas-Fermi-like variational theory of 2D molecules. We consider the functional

$$
\mathcal{E}^{\mathrm{TF}}[\sigma]=-\int_{\mathbb{R}^{2}} V_{\mathrm{nuc}}(x) \sigma(x) d x+D(\sigma, \sigma)
$$

where $\sigma$ is the density distribution of negatively charged "electrons",

$$
V_{\text {nuc }}(x)=-\sum_{i=1}^{K} \log \left|x-x_{i}\right|
$$

is the $2 \mathrm{D}$ electrostatic potential generated by $K$ point "nuclei" of charge +1 located at positions $x_{1}, \ldots, x_{K}$ in the plane and

$$
D(\sigma, \sigma)=-\frac{1}{2} \iint_{\mathbb{R}^{2} \times \mathbb{R}^{2}} \sigma(x) \log |x-y| \sigma(y) d x d y
$$

is the electrostatic self-energy of the "electrons".

We emphasize that in the application we have in mind, the designations "nuclei"and "electrons" do not refer to real physical entities. The terminology is just chosen for convenience and analogy with standard TF theory for molecules [15,40].

We consider the minimization of $\mathcal{E}^{\mathrm{TF}}$ over the variational set

$$
\mathcal{M}^{\mathrm{TF}}:=\left\{\sigma \in L^{\infty}\left(\mathbb{R}^{2}\right) \cap L^{1}\left(\mathbb{R}^{2}, \log (2+|x|) d x\right), 0 \leq \sigma \leq 1, \int_{\mathbb{R}^{2}} \sigma=K\right\}
$$

which means that we are interested in an "incompressible neutral molecule". The term "incompressible" refers to the constraint $\sigma \leq 1$ on the density of electrons and "neutral" to the requirement that the total charge of the electrons equals that of the nuclei. 
Remark 3.1 (Related models). From a physics point of view, a more natural functional to consider would be

$$
\mathcal{E}_{\tau}[\sigma]=\int_{\mathbb{R}^{2}} \tau(\sigma)-\int_{\mathbb{R}^{2}} V_{\text {nuc }}(x) \sigma(x) d x+D(\sigma, \sigma)
$$

with a function $\tau$ representing the kinetic energy density of the electrons. For $\tau(\sigma)=$ (const.) $\cdot \sigma^{2}$ this term is the standard local density/semi-classical approximation of the quantum kinetic energy of a $2 \mathrm{D}$ electron gas. The minimization problem (3.1)-(3.4), which is the relevant one for our purpose, corresponds formally to taking $\tau(\sigma)=\sigma^{p}$ with $p \rightarrow \infty$ to enforce the uniform upper bound $\sigma \leq 1$, but we shall not follow this $p \rightarrow \infty$ route.

Many results we prove below have a natural extension to the case where $V_{\text {nuc }}$ is a more general potential, as long as it is generated by a compactly supported charge distribution. One could also consider corresponding 3D models as e.g. in [12], where results parallel to those of this section have been obtained. Note that the $3 \mathrm{D}$ setting is in fact simpler to deal with, because of the fall-off at infinity of the 3D Coulomb kernel.

We also mention that there is a kinship with recently studied flocking/swarming models $[13,23]$ where constrained minimization problems similar to the above have been considered and proved to support phase transitions.

The facts we shall need about the variational problem for $\mathcal{E}^{\mathrm{TF}}$ are collected in the following theorem.

Theorem 3.2 (Incompressible neutral Thomas-Fermi molecules). Let $K$ be a positive integer. We have

(1) Well-posedness. There exists a unique $\sigma^{\mathrm{TF}}$ that minimizes $\mathcal{E}^{\mathrm{TF}}$ over the variational set $\mathcal{M}^{\mathrm{TF}}$.

(2) Potential and variational inequalities. Let

$$
\Phi^{\mathrm{TF}}:=V_{\mathrm{nuc}}+\sigma^{\mathrm{TF}} * \log |\cdot|
$$

be the total electrostatic potential of the molecule. It is continuous and once continuously differentiable away from the nuclei and tends to zero at infinity. Moreover, for almost all $x$,

$$
\begin{array}{ll}
\Phi^{\mathrm{TF}}(x)>0 & \text { if } \sigma^{\mathrm{TF}}(x)>0 \\
\Phi^{\mathrm{TF}}(x)=0 & \text { if } \sigma^{\mathrm{TF}}(x)=0 .
\end{array}
$$

(3) Values of the electron density. The set $\left\{x: 0<\sigma^{\mathrm{TF}}(x)<1\right\}$ has zero Lebesgue measure. Hence $\sigma^{\mathrm{TF}}$ takes only the values 1 or 0 a.e.

(4) Support of the electron density.

(i) The set $\left\{x: \sigma^{\mathrm{TF}}(x)>0\right\}$ is essentially bounded: If $D(0, r)$ denotes a disk with radius $r$ and center at 0 , then for any $R>\max \left|x_{i}\right|$ we have, up to a set of measure zero,

$$
\left\{x: \sigma^{\mathrm{TF}}(x)>0\right\} \subset D\left(0, R+\sqrt{M_{R}}\right)
$$

with

$$
M_{R}:=\frac{1}{\pi} \sup _{|x|=R} \Phi^{\mathrm{TF}}(x) .
$$


(ii) Up to a set of measure zero, $\left\{x: \sigma^{\mathrm{TF}}(x)>0\right\}$ is equal to the open set

$$
\Sigma^{\mathrm{TF}}\left(x_{1}, \ldots, x_{K}\right)=\left\{x: \Phi^{\mathrm{TF}}(x)>0\right\}
$$

and the area of $\Sigma^{\mathrm{TF}}\left(x_{1}, \ldots, x_{K}\right)$ is equal to $K$.

(iii) The locations $x_{1}, \ldots, x_{K}$ of the nuclei lie within $\Sigma^{\mathrm{TF}}\left(x_{1}, \ldots, x_{K}\right)$ and all connected components of the latter set contain at least one $x_{j}$.

$$
\Sigma^{\mathrm{TF}}\left(x_{1}, \ldots, x_{K-1}\right) \subset \Sigma^{\mathrm{TF}}\left(x_{1}, \ldots, x_{K}\right) .
$$

(v) For a single nucleus,

$$
\Sigma^{\mathrm{TF}}\left(x_{1}\right)=\left\{x:\left|x-x_{1}\right|<1 / \sqrt{\pi}\right\} .
$$

The inclusion in (3.11) says that when a nucleus is added, the density $\sigma^{\mathrm{TF}}$ and the potential $\Phi^{\mathrm{TF}}$ cannot decrease anywhere. The rest of this section is devoted to the proof of these results.

3.1. Existence and uniqueness of a minimizer. The uniqueness is simpler than the existence and we consider it first:

Lemma 3.3 (Strict convexity and uniqueness). The functional $\mathcal{E}^{\mathrm{TF}}$ is strictly convex on $\mathcal{M}^{\mathrm{TF}}$. Consequently, the minimizer $\sigma^{\mathrm{TF}}$, if it exists, is unique.

Proof. We only have to show that $D(\sigma, \sigma)$ is strictly convex on $\mathcal{M}^{\mathrm{TF}}$. This is a consequence of the positivity of $D(\mu, \mu)$ for neutral charge distributions $\mu$ as in [58, Lemma 3.2]. The general fact is that

$$
D(\mu, \mu)=\frac{1}{2 \pi} \int_{\mathbb{R}^{2}}\left(\int_{\mathbb{R}^{2}} \frac{1}{|t-z|} d \mu(z)\right)^{2} d t
$$

whenever $\int_{\mathbb{R}^{2}} \mu=0$, see [62, Chapter I, Lemma 1.8]. This implies the claimed strict convexity property: pick two measures $\mu_{1}$ and $\mu_{2}$ with $\int \mu_{1}=\int \mu_{2}$. Then

$$
\begin{aligned}
& \frac{1}{2} D\left(\mu_{1}, \mu_{1}\right)+\frac{1}{2} D\left(\mu_{2}, \mu_{2}\right)-D\left(\frac{1}{2} \mu_{1}+\frac{1}{2} \mu_{2}, \frac{1}{2} \mu_{1}+\frac{1}{2} \mu_{2}\right) \\
& =\frac{1}{4} D\left(\mu_{1}-\mu_{2}, \mu_{1}-\mu_{2}\right) \geq 0
\end{aligned}
$$

since $\int_{\mathbb{R}^{2}} \mu_{1}=\int_{\mathbb{R}^{2}} \mu_{2}=1$. Equality holds if and only if $\mu_{1}=\mu_{2}$ a.e. Uniqueness of the minimizer follows because we minimize a strictly convex functional on a convex set.

Next we consider the boundedness from below and lower semicontinuity of the functional $\mathcal{E}^{\mathrm{TF}}$.

Lemma 3.4 (Boundedness from below). The functional $\mathcal{E}^{\mathrm{TF}}$ is uniformly bounded from below on $\mathcal{M}^{\mathrm{TF}}$. It is moreover lower semi-continuous under strong convergence in $L^{1} \cap L^{p}$ for any $p>1$. 
Proof. For any $\sigma \in \mathcal{M}^{\mathrm{TF}}$ we can use the normalization to write

$$
\begin{aligned}
\mathcal{E}^{\mathrm{TF}}[\sigma]= & \frac{1}{2} \iint_{\mathbb{R}^{2} \times \mathbb{R}^{2}}\left(K^{-1} \sum_{i=1}^{K} \log \left|x-x_{i}\right|+K^{-1} \sum_{i=1}^{K} \log \left|y-x_{i}\right|-\log |x-y|\right) \\
& \times \sigma(x) \sigma(y) d x d y .
\end{aligned}
$$

Then, since $|x-y| \leq(1+|x|)(1+|y|)$, we have

$$
\mathcal{E}^{\mathrm{TF}}[\sigma] \geq K \int_{\mathbb{R}^{2}}\left(K^{-1} \sum_{i=1}^{K} \log \left|x-x_{i}\right|-\log (1+|x|)\right) \sigma(x) d x .
$$

The function in parenthesis is clearly uniformly bounded from below outside of a disk $D(0, R)$ that contains all the nuclei. On the disk $D(0, R)$ it is locally integrable. Since $\sigma \in \mathcal{M}^{\mathrm{TF}}$ is uniformly bounded in $\in L^{1}\left(\mathbb{R}^{2}\right) \cap L^{\infty}\left(\mathbb{R}^{2}\right)$ and non-negative, we can split the integration domain according to whether $|x| \leq R$ or the other way around, and conclude that both pieces are bounded from below uniformly.

The lower semi-continuity follows from the same kind of considerations, using in addition Fatou's lemma and the fact that $\log |.| \in L_{\text {loc }}^{q}\left(\mathbb{R}^{2}\right)$ for any $1 \leq q<\infty$.

We now prove the existence of a minimizer. Note that if we had been minimizing under the constraint that the mass be equal to $N<K$, existence of a minimizer would follow from standard arguments discussed e.g. in [62]. At neutrality, when the number of electrons equals that of nuclei, this is slightly more subtle and we use some tricks we learned from $[10,27,28]$.

Proof of Theorem 3.2, Item 1. Uniqueness of the minimizer is already contained in Lemma 3.3. For the existence, consider a minimizing sequence $\left(\sigma_{n}\right)_{n \in \mathbb{N}}$. In view of the definition of $\mathcal{M}^{\mathrm{TF}}$ we can extract a (not relabeled) subsequence which converges weakly- $\star$ as a Radon measure, weakly in any $L^{p}\left(\mathbb{R}^{2}\right)$ for any $1<p<\infty$, and weakly- $\star$ in $L^{\infty}\left(\mathbb{R}^{2}\right)$ to a candidate minimizer $\sigma^{\mathrm{TF}}$, i.e.

$$
\int_{\mathbb{R}^{2}} f \sigma_{n} \rightarrow \int_{\mathbb{R}^{2}} f \sigma
$$

for any function $f \in L^{q}\left(\mathbb{R}^{2}\right), 1 \leq q<\infty$ (the convergence as a Radon measure corresponds to $f$ continuous with compact support). Using Mazur's lemma [41, Theorem 2.13] and the convexity of the functional we could assume without loss that the convergence is strong in any $L^{q}\left(\mathbb{R}^{2}\right), 1<q<\infty$, but we shall not use this.

By the lower semicontinuity proved in Lemma 3.4 we obtain

$$
\mathcal{E}^{\mathrm{TF}}\left[\sigma^{\mathrm{TF}}\right] \leq \inf \left\{\mathcal{E}^{\mathrm{TF}}[\sigma]: \sigma \in \mathcal{M}^{\mathrm{TF}}\right\}
$$

Hence we only have to prove that $\sigma^{\mathrm{TF}} \in \mathcal{M}^{\mathrm{TF}}$. The upper and lower constraints on $\sigma_{n}$ pass to the limit easily, but it remains to show that

$$
\int_{\mathbb{R}^{2}} \sigma^{\mathrm{TF}}=K
$$


This follows from the fact that the sequence $\left(\sigma_{n}\right)_{n \in \mathbb{N}}$ is tight, i.e., no mass escapes to infinity in the limit. To prove this we pick some $R>0$ and rewrite the energy as in (3.15), then split the part of the double integral where $|x| \geq R,|y| \geq R$ from the rest:

$$
\begin{aligned}
\mathcal{E}^{\mathrm{TF}}\left[\sigma_{n}\right] & \\
= & \frac{1}{2} \iint_{|x| \geq R,|y| \geq R}\left(K^{-1} \sum_{i=1}^{K} \log \left|x-x_{i}\right|+K^{-1} \sum_{i=1}^{K} \log \left|y-x_{i}\right|-\log |x-y|\right) \\
& \sigma_{n}(x) \sigma_{n}(y) d x d y \\
& +\frac{1}{2} \iint_{|x| \leq R} \text { or }|y| \leq R \\
& \sigma_{n}(x) \sigma_{n}(y) d x d y .
\end{aligned}
$$

The second term is bounded below independently of $n$ and $R$ by the same arguments as in the proof of Lemma 3.4. For the rest we rewrite

$$
\begin{aligned}
& K^{-1} \sum_{i=1}^{K} \log \left|x-x_{i}\right|+K^{-1} \sum_{i=1}^{K} \log \left|y-x_{i}\right|-\log |x-y| \\
& =\left(K^{-1} \sum_{i=1}^{K} \log \left|x-x_{i}\right|-\frac{1}{2} \log \left(1+|x|^{2}\right)+K^{-1} \sum_{i=1}^{K} \log \left|y-x_{i}\right|-\frac{1}{2} \log \left(1+|y|^{2}\right)\right) \\
& \quad-\log \frac{|x-y|}{\sqrt{1+|x|^{2}} \sqrt{1+|y|^{2}}} .
\end{aligned}
$$

The function in parenthesis on the second line is bounded below uniformly for $|x|,|y| \geq$ $R$ large enough, so that, once integrated against $\mathbb{1}_{|x| \geq R} \mathbb{1}_{|y| \geq R} \sigma_{n}(x) \sigma_{n}(y) d x d y$ it gives a contribution uniformly bounded from below. For the contribution of the last term we note that

$$
\frac{|x-y|}{\sqrt{1+|x|^{2}} \sqrt{1+|y|^{2}}} \leq \frac{|x|+|y|}{\sqrt{1+|x|^{2}} \sqrt{1+|y|^{2}}} \leq \frac{1}{\sqrt{1+|y|^{2}}}+\frac{1}{\sqrt{1+|x|^{2}}}
$$

and thus

$$
\begin{aligned}
& \iint_{|x| \geq R,|y| \geq R} \log \frac{|x-y|}{\sqrt{1+|x|^{2}} \sqrt{1+|y|^{2}}} \sigma_{n}(x) \sigma_{n}(y) d x d y \\
& \leq-\frac{1}{2} \log \left(1+R^{2}\right)\left(\int_{|x| \geq R} \sigma_{n}(x) d x\right)^{2}+C .
\end{aligned}
$$

All in all we deduce that, the energy of $\sigma_{n}$ being uniformly bounded above,

$$
\frac{1}{4} \log \left(1+R^{2}\right)\left(\int_{|x| \geq R} \sigma_{n}(x) d x\right)^{2} \leq C,
$$

where $C$ depends neither on $n$ nor on $R>0$ provided the latter is large enough. Since $\log \left(1+R^{2}\right) \rightarrow+\infty$ for $R \rightarrow \infty$, this implies tightness of the minimizing sequence, namely that for any $\varepsilon>0$ there exists $R_{\varepsilon}>0$ such that 


$$
\limsup _{n \rightarrow \infty} \int_{|x| \geq R_{\varepsilon}} \sigma_{n}(x) d x<\varepsilon
$$

To study the properties of the minimizer, whose existence and uniqueness we have now established, we next consider the corresponding electrostatic potential.

3.2. The Thomas-Fermi potential. To any electron density $\sigma$ we associate a potential

$$
\Phi_{\sigma}=V_{\text {nuc }}+\sigma * \log |.|=\left(\rho_{\text {nuc }}-\sigma\right) *(-\log |\cdot|) .
$$

If $\int_{\mathbb{R}^{2}} \sigma=K$ then this is the Coulomb potential generated by a neutral charge distribution. We prove first that it is regular away from the nuclei and decays at infinity.

Lemma 3.5 (Regularity and asymptotic behavior of the potential). If $\sigma \in \mathcal{M}^{\mathrm{TF}}$, the potential $\Phi_{\sigma}$ is continuous and once continuously differentiable away from the nuclei and tends to zero at infinity. More generally, if $\sigma$ satisfies the conditions (3.4) except neutrality, but $\int \sigma<K$, then

$$
\left|\Phi_{\sigma}(x)+\left(K-\int_{\mathbb{R}^{2}} \sigma\right) \log \right| x|| \rightarrow 0
$$

when $|x| \rightarrow \infty$

Proof. If $\sigma \in \mathcal{M}^{\mathrm{TF}}$ has compact support the regularity follows from [41, Theorem 10.2]. The general case follow by cutting $\sigma$ at some finite radius $R$ and using the dominated convergence theorem as $R \rightarrow \infty$.

For the asymptotic behavior we consider also first the case that $\sigma$ has support in some disk $D(0, R)$ with $R<\infty$. For $|y| \leq \frac{1}{2}|x|$ we have

$$
\log |x-y|=\log |x|+\log \left|1-\frac{y}{x}\right| \leq \log |x|+\frac{|y / x|}{1-|y / x|} \leq \log |x|+2 \frac{|y|}{|x|}
$$

where the ratio of two vectors in $\mathbb{R}^{2}$ is interpreted as that of the corresponding complex numbers.

Thus, for $|x| \geq 2 \max _{i}\left\{R,\left|x_{i}\right|\right\}$

$$
\left|\Phi_{\sigma}(x)+\left(K-\int_{\mathbb{R}^{2}} \sigma\right) \log \right| x|| \leq 2\left(\sum_{i=1}^{K}\left|x_{i}\right|+\int_{D(0, R)}|y| \sigma(y) d y\right) \frac{1}{|x|} .
$$

and the result is proved in this case.

Let us now only assume that

$$
\int_{\mathbb{R}^{2}} \sigma(y) \log (1+|y|) d y<\infty
$$

as in the definition of $\mathcal{M}^{\mathrm{TF}}$. This implies that for any $\varepsilon>0$ there is a $R_{\varepsilon}$ such that

$$
\int_{|y| \geq R_{\varepsilon}} \sigma(y) \log (1+|y|) d y<\varepsilon .
$$


For every $\varepsilon>0$ there is a $\delta>0$ such that $\log (1-t)<\varepsilon$ if $|t|<\delta$. Pick some large $R$ and write for $|x| \geq R \geq R_{\varepsilon}$

$$
\int_{\mathbb{R}^{2}} \log \left|1-\frac{y}{x}\right| \sigma(y) d y=\int_{|y| \leq \delta \cdot R} \log \left|1-\frac{y}{x}\right| \sigma(y) d y+\int_{|y| \geq \delta \cdot R} \log \left|1-\frac{y}{x}\right| \sigma(y) d y .
$$

The first term is bounded by (const.) $\varepsilon$ by the choice of $\delta$ and because $\int_{\mathbb{R}^{2}} \sigma$ is finite.

The second term we write as

$$
\int_{|y| \geq \delta \cdot R} \log |x-y| \sigma(y) d y-\log |x| \int_{|y| \geq \delta \cdot R} \sigma(y) d y .
$$

If $\delta \cdot R$ is large enough, we can ensure

$$
\int_{|y| \geq \delta \cdot R} \sigma(y) d y \leq C \varepsilon(\log |x|)^{-1} .
$$

because of (3.31). In the remaining term (remember that $\delta \cdot R$ is large and $|x| \geq \delta \cdot R$ ) we split the integral into a disk $D(x, 1)$ of radius 1 around $x$ and the rest. Since the logarithm is square integrable over the unit disk we can use the Cauchy-Schwarz inequality to conclude that

$$
\left|\int_{D(x, 1)} \log \right| x-y|\sigma(y) d y| \leq C \varepsilon^{1 / 2}
$$

because $0 \leq \sigma \leq 1$ and $\int_{D(x, 1)} \sigma \leq \varepsilon$. The integral over the complement of $D(x, 1)$ is also small because there $\log |x-y| \leq 2 \log |y|$.

Summarizing, we have proved that for any $\varepsilon>0$ there exists $R_{\varepsilon}>0$ such that

$$
\int_{\mathbb{R}^{2}} \log \left|1-\frac{y}{x}\right| \sigma(y) d y \leq \varepsilon
$$

for $|x| \geq R_{\varepsilon}$. Returning to the first equality in (3.28) proves the lemma.

Lemma 3.6 (Variational inequalities). Recall the definition (3.6) of the Thomas-Fermi potential. There exists $\lambda \in \mathbb{R}$ such that for almost every $x$

$$
\begin{aligned}
& \Phi^{\mathrm{TF}}(x) \geq \lambda \text { if } \sigma^{\mathrm{TF}}(x)=1 \\
& \Phi^{\mathrm{TF}}(x)=\lambda \text { if } 0<\sigma^{\mathrm{TF}}(x)<1 \\
& \Phi^{\mathrm{TF}}(x) \leq \lambda \text { if } \sigma^{\mathrm{TF}}(x)=0
\end{aligned}
$$

Proof. Equations (3.37)-(3.39) follow from local minimality of the functional (3.1) at $\sigma^{\mathrm{TF}}$ by performing small variations in the standard way, c.f. [24, Lemma 4.1.2] or $[13,23]$. Here $\lambda$ is the Lagrange multiplier associated with the mass constraint. That we get only inequalities on the sets $\left\{\sigma^{\mathrm{TF}}=1\right\}$ and $\left\{\sigma^{\mathrm{TF}}=0\right\}$ comes from the fact that the variational constraint $0 \leq \sigma \leq 1$ allows only one-sided variations on these sets.

The Lagrange multiplier $\lambda$ we shall refer to as a chemical potential for it is related ${ }^{5}$ to variations of the minimal energy as a function of the electronic charge. We have the following:

\footnotetext{
5 We do not make this relation explicit for we don't need it here. See [44] where this is explained in the setting of usual Thomas-Fermi theory.
} 
Lemma 3.7 (Value of the chemical potential). The Lagrange multiplier $\lambda$ defined in Lemma 3.6 is equal to 0 and $\Phi^{\mathrm{TF}}=0$ a.e. where $\sigma^{\mathrm{TF}}=0$.

Proof. Assume $\lambda<0$. By Lemma $3.5 \Phi^{\mathrm{TF}}$ vanishes at infinity so there is a radius $R$ such that $\Phi^{\mathrm{TF}}(x)>\lambda$ for $|x| \geq R$. By (3.37)-(3.39) this implies $\sigma^{\mathrm{TF}}(x)=1$ a.e. for $|x| \geq R$, contradicting the finiteness of $\int \sigma$. Hence $\lambda<0$ is excluded.

Assume $\lambda>0$. As above, there is a radius $R$ such that $\Phi^{\mathrm{TF}}(x)<\lambda$ for $|x| \geq R$. By (3.37)-(3.39) this implies $\sigma^{\mathrm{TF}}(x)=0$ a.e. for $|x| \geq R$. Hence the set $\left\{\sigma^{\mathrm{TF}}>0\right\}$ is bounded, up to a set of measure zero. Consider the circular average

$$
\bar{\Phi}(R):=\frac{1}{2 \pi} \int_{0}^{2 \pi} \Phi^{\mathrm{TF}}\left(R e^{i \theta}\right) d \theta .
$$

Because $\Phi^{\mathrm{TF}}$ is harmonic on $\left\{x: \Phi^{\mathrm{TF}}(x)<\lambda\right\}$, it cannot have a local minimum on this set so we know that $\Phi^{\mathrm{TF}}(x) \geq 0$ everywhere and hence $\bar{\Phi}(R) \geq 0$. By continuity of $\Phi^{\mathrm{TF}}$ and the assumption $\lambda>0$ there is a radius $R_{0}$ such that $\bar{\Phi}\left(R_{0}\right)>0$, but $\bar{\Phi}(R)<\lambda$ for $R>R_{0}$. The circle with radius $R_{0}$ thus encloses the whole set $\left\{\sigma^{\mathrm{TF}}>0\right\}$, up to a set of measure zero. On the other hand, by Newton's Theorem [41, Theorem 9.7], $\bar{\Phi}\left(R_{0}\right)$ is proportional to the total charge inside the circle of radius $R_{0}$ which is zero and we have a contradiction.

Having excluded both $\lambda<0$ and $\lambda>0$ we conclude that $\lambda=0$. Finally, $\{x$ : $\left.\Phi^{\mathrm{TF}}(x)<0\right\}$ is empty because $\Phi^{\mathrm{TF}}$ would be harmonic on this set and zero on its boundary.

With Lemmas 3.6 and 3.7 we have established Item (2) of Theorem 3.2 We next show that $\sigma^{\mathrm{TF}}$ takes only the values 0 or 1 almost everywhere.

Proof of Theorem 3.2, Item (3). We want to show that the set

$$
A:=\left\{x: 0<\sigma^{\mathrm{TF}}(x)<1\right\}
$$

has zero Lebesgue measure. By the definition (3.6) of $\Phi^{\mathrm{TF}}$ we have in the sense of distributions

$$
-\Delta \Phi^{\mathrm{TF}}=2 \pi\left(\rho_{\text {nuc }}-\sigma^{\mathrm{TF}}\right) .
$$

with $\rho_{\text {nuc }}=\sum_{i=1}^{K} \delta_{x_{i}}$. Because $\Phi^{\mathrm{TF}}$ is constant (in fact zero) on $A$, we can immediately exclude that $A$ contains an open set, because on such a set we would have

$$
\Delta \Phi^{\mathrm{TF}}=0
$$

contradicting (3.42). Since there exist sets of positive measure but with empty interior this does not prove the lemma, however. The problem of overcoming the lack of knowledge of $A$, and showing that (3.43) holds a.e. on $A$ was solved in a similar problem in [23] and we sketch that argument here.

If a function $f$ is in $W_{\text {loc }}^{1,1}$ then on the set on which $f$ has any fixed value, its gradient, $\nabla f$, vanishes except for a set of measure zero, see [1,46,68] or [41, Theorem 6.9]. Away from the nuclei, $\Phi^{\mathrm{TF}}$ is explicitly seen to be continuous and in $L_{\mathrm{loc}}^{1}$, and the same holds for its gradient $\nabla \Phi^{\mathrm{TF}}$. On $A$ we thus have $\nabla \Phi^{\mathrm{TF}}=0$. In order to repeat this argument for the Laplacian, we need to show that $\Phi^{\mathrm{TF}}$ is in $W_{\text {loc }}^{2,1}$. 
What we do know is that the sum of the second derivatives, $\Delta \Phi^{\mathrm{TF}}$, is an $L_{\text {loc }}^{1}$ function (see (3.42)). What we need is that all mixed second derivatives are in $L_{\text {loc }}^{1}$. This follows by an exercise involving Fourier transforms, c.f. [23, Lemma 11]. Thus (3.43) does hold a.e. on $A$, and this contradicts (3.42) unless $A$ has zero Lebesgue measure.

We now turn to the last item of Theorem 3.2, concerning the support of the electron density.

\section{Proof of Theorem 3.2, Item (4).}

Step 1. We first prove the estimate (3.8) on the support of $\sigma^{\mathrm{TF}}$. By Lemma 3.7 we know that $\lambda=0$. There is an $R$ such that $\left|x_{i}\right|<R$ for all $i$. For $|x| \geq R$ and $\hat{R}>R$ we introduce the function

$$
g(x)=\left\{\begin{array}{ll}
\pi \cdot(\hat{R}-|x|)^{2} & \text { if }|x| \leq \hat{R} \\
0 & \text { if }|x|>\hat{R}
\end{array} .\right.
$$

Then, for $R<|x| \leq \hat{R}$,

$$
\Delta g \leq 2 \pi
$$

Consider the set

$$
\mathcal{S}=\left\{x: 0 \leq g(x)<\Phi^{\mathrm{TF}}(x),|x| \geq R\right\} .
$$

Since $\Phi^{\mathrm{TF}}>0$ on this set, which contains no nucleus by assumption, we have $\sigma^{\mathrm{TF}}=1$ and $\Delta \Phi^{\mathrm{TF}}=2 \pi$ on $\mathcal{S}$. Hence

$$
\Delta\left(g-\Phi^{\mathrm{TF}}\right) \leq 0
$$

on $\mathcal{S}$, so the function $g-\Phi^{\mathrm{TF}}$ is superharmonic on $\mathcal{S}$ and reaches its minimum at the boundary or at infinity. At infinity the function vanishes. For $|x|=R$ we can make $g-\Phi^{\mathrm{TF}} \geq 0$ by choosing

$$
\hat{R}=R+\sqrt{M_{R}}
$$

where $M_{R}$ is given by (3.9). We conclude that on $\mathcal{S}$ we have $g-\Phi^{\mathrm{TF}} \geq 0$ and thus this set must be empty. Then $\Phi^{\mathrm{TF}} \leq g$ for $|x| \geq R$, so $\Phi^{\mathrm{TF}}=0$ and hence $\sigma^{\mathrm{TF}}=$ $(2 \pi)^{-1} \Delta \Phi^{\mathrm{TF}}=0$ for $|x|>\hat{R}$. This yields the desired result, Eq. (3.8).

Step 2. Next we consider the open set $\Sigma^{\mathrm{TF}}\left(x_{1}, \ldots, x_{K}\right) \equiv \Sigma^{\mathrm{TF}}$ where the potential $\Phi^{\mathrm{TF}}$ is strictly positive. From Eq. (3.7) we have $\Sigma^{\mathrm{TF}} \subset\left\{\sigma^{\mathrm{TF}}>0\right\}$ while $\left\{\sigma^{\mathrm{TF}}>0\right\}$ is contained in the closure $\bar{\Sigma}^{\mathrm{TF}}$. Since $\Phi^{\mathrm{TF}}$ is continuous outside the "nuclei", $\Sigma^{\mathrm{TF}}$ is Jordan measurable which means that its boundary has Lebesgue measure zero. Hence $\Sigma^{\mathrm{TF}}$ and $\left\{\sigma^{\mathrm{TF}}>0\right\}$ are equal up to a null set. Since $\sigma^{\mathrm{TF}}$ takes only the values 1 or 0 , it follows that the area of $\Sigma^{\mathrm{TF}}$ is equal to $K$.

Step 3. Since $\Phi^{\mathrm{TF}}(x) \rightarrow+\infty$ when $x$ approaches a location of a nucleus, we have $x_{i} \in \Sigma^{\mathrm{TF}}$ for all $i$. Consider a connected component of $\Sigma^{\mathrm{TF}}$ which does not include any $x_{i}$. On this component we have

$$
\Delta \Phi^{\mathrm{TF}}=2 \pi
$$

and thus $\Phi^{\mathrm{TF}}$ is subharmonic, hence reaches its maximum at the boundary, where $\Phi^{\mathrm{TF}}=$ 0 . This contradicts the strict positivity of $\Phi^{\mathrm{TF}}$ on $\Sigma^{\mathrm{TF}}\left(x_{1}, \ldots, x_{K}\right)$. Hence all connected components of $\Sigma^{\mathrm{TF}}$ contain at least one nucleus.

Step 4. Denote by $\Phi_{K-1}^{\mathrm{TF}}$ and $\Phi_{K}^{\mathrm{TF}}$ the TF potentials corresponding to $\left\{x_{1}, \ldots, x_{K-1}\right\}$ and $\left\{x_{1}, \ldots, x_{K}\right\}$ respectively and by $\sigma_{K}$ and $\sigma_{K-1}$ the minimizing densities. Likewise 
we denote by $\Sigma_{K-1}^{\mathrm{TF}}$ and $\Sigma_{K}^{\mathrm{TF}}$ the sets where the potentials $\Phi_{K-1}^{\mathrm{TF}}$ and $\Phi_{K}^{\mathrm{TF}}$ are strictly positive. Consider the open set

$$
\mathcal{U}=\left\{x: \Phi_{K}^{\mathrm{TF}}(x)<\Phi_{K-1}^{\mathrm{TF}}\right\} .
$$

Clearly, $x_{i} \notin \mathcal{U}$ for all $i=1, \ldots, K$. Since $\Phi_{K}^{\mathrm{TF}} \geq 0$ we have $\Phi_{K-1}>0$ on $\mathcal{U}$ and hence $\sigma_{K-1}=1$ on $\mathcal{U}$. Thus

$$
(2 \pi)^{-1} \Delta\left(\Phi_{K-1}-\Phi_{K}\right)=\sigma_{K-1}-\sigma_{K} \geq 0
$$

on $\mathcal{U}$ because $\sigma_{K} \leq 1$. Thus $\Phi_{K-1}-\Phi_{K}$ is subharmonic on $\mathcal{U}$ and $\mathcal{U}$ must be empty. We conclude that

$$
\Phi_{K-1}(z) \leq \Phi_{K}(z)
$$

everywhere. In $\Sigma_{K-1}^{\mathrm{TF}}$ we have $\Phi_{K-1}(z)>0$, hence $\Phi_{K}(z)>0$, so $\Sigma_{K-1}^{\mathrm{TF}} \subset \Sigma_{K}^{\mathrm{TF}}$.

Step 5. For a single nucleus, uniqueness implies that the minimizer must be rotationally symmetric around $x_{1}$. By Step 3 the support of the density is connected and hence a full disk with $\sigma^{\mathrm{TF}}=1$. The Poisson equation then lead to the unique solution

$$
\Phi^{\mathrm{TF}}(x)=\left\{\begin{array}{ll}
-\log \left|x-x_{1}\right|+\pi\left|x-x_{1}\right|^{2}-\frac{1}{2} \log \pi-1 & \text { if }\left|x-x_{1}\right|<1 / \sqrt{\pi} \\
0 & \text { if }\left|x-x_{1}\right| \geq 1 / \sqrt{\pi}
\end{array} .\right.
$$

\section{Local Density Upper Bounds for Classical Ground States}

In this section we discuss the ground state of a generalized classical jellium Hamiltonian as presented in the introduction, and prove the announced local density upper bound on any microscopic scale $\gg 1$. The argument is split in two steps, corresponding to Sects. 4.1 and 4.2:

- First we prove an exclusion rule for ground states configurations: no point can lie inside a screening region defined by any subset of the other points. The latter is given by the open set $\Sigma^{\mathrm{TF}}$ where the TF potential $\Phi^{\mathrm{TF}}$ corresponding to the chosen subset of the ground state configuration is strictly positive.

- Second, we prove that any configuration of points satisfying the above exclusion rule must have a local density bounded above by 1 , which is the desired optimal upper bound.

In Sect. 4.3 we apply these results to ground states of the Hamiltonian (2.16) entering the plasma analogy, and small perturbations thereof.

4.1. Exclusion by screening. The exclusion rule we shall chiefly rely on in the sequel is as follows:

Definition 4.1 (Exclusion by screening). Let $X_{N}=\left\{x_{1}, \ldots, x_{N}\right\} \subset \mathbb{R}^{2}$. Take any subset $\left\{y_{1}, \ldots, y_{K}, y_{K+1}\right\} \subset X_{N}$ of distinct points $y_{i}$. Define as in (3.10) $\Sigma^{\mathrm{TF}}\left(y_{1}, \ldots, y_{K}\right)$ to be the open set where the TF potential (3.6), generated by nuclei at locations $y_{1}, \ldots, y_{K}$ and the corresponding background charge distribution $\sigma^{\mathrm{TF}}$, is strictly positive. We say that $X_{N}$ satisfies the exclusion rule if

$$
y_{K+1} \notin \Sigma^{\mathrm{TF}}\left(y_{1}, \ldots, y_{K}\right)
$$

holds for any choice of $y_{1}, \ldots, y_{K}, y_{K+1} \in X_{N}$. 
We call this "exclusion by screening" because $\Sigma^{\mathrm{TF}}$ defines a region surrounding $y_{1}, \ldots, y_{K}$ outside of which the background charge density $\sigma^{\mathrm{TF}}$ totally screens the potential generated by $y_{1}, \ldots, y_{K}$. Indeed, as we proved in the preceding section, Eq. (3.7), the total potential generated by the background and the point charges together vanishes identically outside $\Sigma^{\mathrm{TF}}$. Note also that the closure of $\Sigma^{\mathrm{TF}}$ is the essential support of $\sigma^{\mathrm{TF}}$.

The relevance of this notion to our problem is as follows: Consider a Hamiltonian function on $\mathbb{R}^{2 N}$ of the form

$$
\mathcal{H}\left(x_{1}, \ldots, x_{N}\right)=\frac{\pi}{2} \sum_{i=1}^{N}\left|x_{i}\right|^{2}-\sum_{1 \leq i<j \leq N} \log \left|x_{i}-x_{j}\right|+\mathcal{W}\left(x_{1}, \ldots, x_{N}\right)
$$

with $\mathcal{W}$ symmetric and superharmonic in each variable $x_{i}$. Equation (4.2) is obtained from (2.16) by a simple change of length units that we perform in order that the maximum local density be 1 instead of $\pi \ell$ (see Sect. 4.3 below).

Proposition 4.2 (Classical ground states satisfy the exclusion rule). Any minimizing configuration $\left\{x_{1}^{0}, \ldots, x_{N}^{0}\right\}$ for $\mathcal{H}$ satisfies the exclusion rule (4.1).

Proof. By symmetry of the Hamiltonian we may, without loss, choose

$$
y_{i}=x_{i}^{0}, 1 \leq i \leq K+1 \text {. }
$$

Consider fixing all points but $x_{K+1}^{0}$. The energy to consider is then

$$
G(x)=\mathcal{H}\left(x_{1}^{0}, \ldots, x_{K}^{0}, x, x_{K+2}^{0}, \ldots x_{N}^{0}\right) .
$$

We claim that if $x \in \Sigma^{\mathrm{TF}}\left(x_{1}^{0}, \ldots, x_{K}^{0}\right) \equiv \Sigma^{\mathrm{TF}}$ then there is an $\tilde{x} \in \partial \Sigma^{\mathrm{TF}}$ such that $G(\tilde{x})<G(x)$. Thus the minimizing point $x_{K+1}^{0}$ cannot lie in $\Sigma^{\mathrm{TF}}$.

To prove the claim, we add and subtract a term $-\int_{\Sigma^{\mathrm{TF}}} \log \left|x-x^{\prime}\right| d x^{\prime}$ to write

$$
\begin{aligned}
& G(x)=\Phi(x)+R(x) \\
& \Phi(x)=-\sum_{i=1}^{K} \log \left|x-x_{i}^{0}\right|+\int_{\Sigma^{\mathrm{TF}}} \log \left|x-x^{\prime}\right| d x^{\prime} \\
& R(x)=\frac{\pi}{2}|x|^{2}-\int_{\Sigma^{\mathrm{TF}}} \log \left|x-x^{\prime}\right| d x^{\prime}-\sum_{i=K+2}^{N} \log \left|x-x_{i}^{0}\right|+\mathcal{W}(x)+\text { const. }
\end{aligned}
$$

with a superharmonic function

$$
\mathcal{W}(x):=\mathcal{W}\left(x_{1}^{0}, \ldots, x_{K}^{0}, x, x_{K+2}^{0}, \ldots x_{N}^{0}\right) .
$$

Now, $\Phi$ is precisely the TF potential corresponding to "nuclear charges" at $x_{i}^{0}, \ldots x_{K}^{0}$. Hence, using (3.7), $\Phi>0$ on $\Sigma^{\mathrm{TF}}$ and zero on the boundary $\partial \Sigma^{\mathrm{TF}}$. The first two terms in $R$ are harmonic on $\Sigma^{\mathrm{TF}}$ when taken together. (The Laplacian applied to the first term gives $2 \pi$ and to the second term $-2 \pi$ on $\Sigma^{\mathrm{TF}}$.) The other terms are superharmonic on $\Sigma^{\mathrm{TF}}$. Thus, $R$ takes its minimum on the boundary, so there is a $\tilde{x} \in \partial \Sigma^{\mathrm{TF}}$ with $R(x) \geq R(\tilde{x})$ for all $x \in \Sigma^{\mathrm{TF}}$. On the other hand, $\Phi(x)>0=\Phi(\tilde{x})$ so $G(x)>G(\tilde{x})$. 
4.2. Exclusion rule and density bounds. We now show that any configuration of points satisfying the exclusion rule (4.1) has its local density everywhere bounded above by 1 . To this end let $R>0$ and define $n(R)$ to be the maximum number of nuclei that a disk $D(a, R)$ of radius $R$ can accommodate while respecting the exclusion rule:

$$
n(R)=\sup \left\{N \in \mathbb{N} \mid \text { there exists } X_{N}=\left\{x_{1}, \ldots, x_{N}\right\} \subset D(a, R) \text { satisfying (4.1) }\right\} \text {. }
$$

It is clear that $n(R)$ is independent of $a$ because a translation of the $x_{i}$ just translates the corresponding exclusion region.

Our density bound is as follows:

Theorem 4.3 (Exclusion rule implies a density bound). We have

$$
\varrho_{\max }:=\limsup _{R \rightarrow \infty} \frac{n(R)}{\pi R^{2}} \leq 1 .
$$

We first recall from [60] a simpler density bound based on an unpublished theorem of Lieb which is essentially (4.1) for the special case $K=1$.

Lemma 4.4 (Minimal distance between points).

Let $\left\{x_{1}, \ldots, x_{N}\right\}$ be a configuration of points satisfying (4.1). Then

$$
\min _{1 \leq i \neq j \leq N}\left|x_{i}-x_{j}\right| \geq \frac{1}{\sqrt{\pi}}
$$

Consequently

$$
\varrho_{\max }=\limsup _{R \rightarrow \infty} \frac{n(R)}{\pi R^{2}} \leq 4
$$

Proof. By Theorem 3.2, Item 4(v), the exclusion region $\Sigma^{\mathrm{TF}}(x)$ for a single point $x$ is the open disk of radius $1 / \sqrt{\pi}$ centered at $x$. Hence (4.11) follows from (4.1).

To deduce (4.12), pick a configuration of points $\left\{x_{1}, \ldots, x_{N}\right\}$ contained in a disk of radius $R$ and smear each point over an open disk of radius $1 /(2 \sqrt{\pi})$. Because of (4.11) these disks do not overlap and must all be contained in a slightly larger disk of radius $R+O(1)$ for large $R$. Thus

$$
\frac{N}{4} \leq \pi R^{2}+O(R)
$$

where the left side is the area covered by the small disks and the right-hand side the area of the large disk. This gives the desired result.

The rough bound Equation (4.12) turns out to be quite useful for the proof of the full Theorem 4.3. The first step is a lemma saying that the lim sup in (4.10) is attained by sequences of configurations with no large vacancies:

Lemma 4.5 (Maximal configurations have no vacancies). Let $R_{k}$ be a sequence of radii with $R_{k} \rightarrow \infty$ for $k \rightarrow \infty$ and $X_{N_{k}}=\left\{x_{1}, \ldots, x_{N_{k}}\right\} \subset D\left(0, R_{k}\right)$ a sequence of configurations such that

$$
\frac{N_{k}}{\pi R_{k}^{2}} \underset{k \rightarrow \infty}{\rightarrow} \varrho_{\max } .
$$

Then, for any fixed $\varepsilon>0$ and any sub-disk $D\left(a, \varepsilon R_{k}\right) \subset D\left(0, R_{k}\right)$,

$$
\sharp\left\{x \in X_{N_{k}} \cap D\left(a, \varepsilon R_{k}\right)\right\} \geq \varrho_{\max } \pi \varepsilon^{2} R_{k}^{2}(1+o(1))
$$

with $o(1) \rightarrow 0$ for $R_{k} \rightarrow \infty$. 
This means that, for a configuration achieving the tightest packing in a given sequence of balls, the density is asymptotically uniform on any length scale comparable to the size of the balls.

Proof. The main idea is that a density lower than $\varrho_{\max }$ in some sub-disk would have to be compensated for by a density higher than $\varrho_{\max }$ in another sub-disk. This would contradict the definition of $\varrho_{\max }$ as the lim sup of the maximal density achievable in any sequence of disks with radii tending to $\infty$.

Note first that by Lemma 4.4 we have for any $R$, as in (4.13),

$$
n(R) \leq \max \left(1,4 \pi R^{2}+C R\right)
$$

Hence $\varrho_{\max }$ is finite and, in fact,

$$
\varrho_{\max } \leq 4
$$

We now tile the disk of radius $R_{k}$ with disjoint smaller disks of radius $s:=\varepsilon R_{k}<1$, labeled by an index $i=1 \ldots I$. We can achieve this leaving only an area $O\left(\varepsilon^{2} R_{k}^{2}\right)$ untiled. The latter we tile with disjoint still smaller disks of radius $t:=\varepsilon^{1 / 2} R_{k}^{1 / 2}$, labeled by $j=1 \ldots J$. In this way we leave only a domain $A_{\mathrm{h}}$ with area $\left|A_{\mathrm{h}}\right|=O\left(\varepsilon R_{k}\right)$ uncovered. Let $n(i, s)$, respectively $n(j, t)$, be the number of points of the configuration $X_{N_{k}}$ in each of the the smaller and the still smaller disks and let $n_{\mathrm{h}}$ be the number of points in $A_{\mathrm{h}}$. By definition of $\varrho_{\max }$ we have

$$
\begin{aligned}
n\left(R_{k}\right)= & \sum_{i=1}^{I} n(i, s)+\sum_{j=1}^{J} n(j, t)+n_{\mathrm{h}} \\
\leq & n(1, s)+\sum_{i=2}^{I} \varrho_{\max } \pi \varepsilon^{2} R_{k}^{2}(1+o(1))+\sum_{j=1}^{J} \varrho_{\max } \pi \varepsilon R_{k}(1+o(1)) \\
& +C \max \left(1,\left|A_{\mathrm{h}}\right|\right) \\
= & n(1, s)+\varrho_{\max }(1+o(1))\left(\pi R_{k}^{2}-\pi \varepsilon^{2} R_{k}^{2}-\left|A_{\mathrm{h}}\right|\right)+C \max \left(1,\left|A_{\mathrm{h}}\right|\right) .
\end{aligned}
$$

In the second line we used the definition of $\varrho_{\max }$ as a lim sup and that for each fixed $\varepsilon>0, \varepsilon R_{k} \rightarrow \infty$. Moreover, we used Lemma 4.4 to bound the density in $A_{\mathrm{h}}$. In the third line we simply used that we tile with disjoint sets. Since by assumption

$$
n\left(R_{k}\right)=\varrho_{\max } \pi R_{k}^{2}(1+o(1))
$$

we deduce

$$
n(1, s) \geq \varrho_{\max } \pi \varepsilon^{2} R_{k}^{2}(1+o(1))\left(1-C \varepsilon^{-1} R_{k}^{-1}\right)+o(1) R_{k}^{2} .
$$

For large $R_{k}$ and fixed $\varepsilon>0$, the small disk labeled $i=1$ must thus satisfy (4.15). For any disk as in the statement, we can construct a tiling such that it is the first small disk and the result follows. 
We now describe briefly the main arguments leading to the proof of Theorem 4.3. The area of the "neutralizing region" $\Sigma^{\mathrm{TF}}$ associated with the nuclear charges contained in a disk of radius $R$ is equal to the number of charges in the disk, so the task is to show that this area is asymptotically equal to the area of the disk as $R \rightarrow \infty$. On the boundary of the region $\Sigma^{\mathrm{TF}}$ the potential generated by the point charges in the disk and the TF neutralizing density $\sigma^{\mathrm{TF}}$ must vanish because of the exclusion rule. In particular, the potential must vanish at any point of the nuclear charge configuration outside the disk. By the previous lemma, we can assume that the density of such vanishing points is bounded below uniformly, and combining this with a bound on the gradient of the potential we obtain an upper bound on the potential outside the disk. We can now proceed in two different ways (the second one was sketched in [42]):

- The bound on the potential outside the disk together with (3.8) implies that we can enclose $\Sigma^{\mathrm{TF}}$ completely in a slightly larger disk whose radius behaves as $R(1+o(1))$. Thus the area of $\Sigma^{\mathrm{TF}}$, which is equal to the number of nuclei in the disk increases at most like the area of the disk plus a small correction.

- Using Newton's theorem we obtain a bound on the circular average of the potential which involves the deviation from neutrality for the charge distribution within the disk. A comparison with the previous upper bound again leads to the desired result: Asymptotically the positive charge cannot be larger than the negative charge in the disk.

The details are as follows, the first steps being common to both arguments.

Proof of Theorem 4.3. Consider any maximal density sequence of radii and configurations defined in the previous Lemma 4.5. We use the lemma in two ways. First, taking $\varepsilon=1 / 2$, we conclude that the sequence of radii $r_{k}:=R_{k} / 2$ and the corresponding number $n_{k}$ of points $\left\{x_{1}, \ldots, x_{n_{k}}\right\}$ in $X_{N_{k}} \cap D\left(0, r_{k}\right)$ satisfy

$$
\lim _{k \rightarrow \infty} \frac{n_{k}}{\pi r_{k}^{2}}=\varrho_{\max }
$$

Second, for every $\varepsilon>0$, the lemma implies that every point in the annulus

$$
\mathcal{A}:=D\left(0, R_{k}\right) \backslash D\left(0, r_{k}\right)
$$

is at most a distance $O\left(\varepsilon r_{k}\right)$ from some point in $X_{N_{k}} \cap \mathcal{A}$ as $r_{k} \rightarrow \infty$.

Let $\sigma_{k}^{\mathrm{TF}}$ be the TF density defined by the point charges $\left\{x_{1}, \ldots, x_{n_{k}}\right\} \subset D\left(0, r_{k}\right)$ and

$$
\Phi_{k}^{\mathrm{TF}}=\left(\sum_{j=1}^{n_{k}} \delta_{x_{j}}-\sigma_{k}^{\mathrm{TF}}\right) *(-\log |.|)
$$

the corresponding TF potential. From Prop. 4.2 we know that $\Phi_{k}^{\mathrm{TF}}$ vanishes at all points in $X_{N_{k}} \cap \mathcal{A}$. Moreover, we know that

$$
\int_{\Sigma_{k}^{T F}} \sigma_{k}^{\mathrm{TF}}=n_{k}
$$

Pick some $\frac{1}{2}>\delta>0$. For $|x| \geq(1+\delta) r_{k}$ a simple estimate gives a bound on the gradient of the potential:

$$
\left|\nabla \Phi_{k}^{\mathrm{TF}}\right| \leq C \frac{n_{k}}{\delta \cdot r_{k}} .
$$


Indeed, if $|x| \geq(1+\delta) r_{k}$ then $\left|x-x_{j}\right| \geq \delta \cdot r_{k}$ for $j=1, \ldots, n_{k}$ and thus

$$
\sum_{j=1}^{n_{k}} \frac{1}{\left|x-x_{j}\right|} \leq \frac{n_{k}}{\delta \cdot r_{k}}
$$

Also, splitting the integral into two regions where $|x-y| \geq \alpha=\sqrt{n_{k}}$ or vice-versa,

$$
\begin{aligned}
\int_{y \in \mathbb{R}^{2}} \frac{1}{|x-y|} \sigma_{k}^{\mathrm{TF}}(y) d y & \leq \int_{|x-y| \leq \alpha} \frac{1}{|x-y|} d y+\alpha^{-1} \int_{\mathbb{R}^{2}} \sigma_{k}^{\mathrm{TF}} \\
& \leq C \alpha+n_{k} \alpha^{-1} \leq C \sqrt{n_{k}} \leq C \frac{n_{k}}{\delta \cdot r_{k}}
\end{aligned}
$$

because $\int \sigma_{k}^{\mathrm{TF}}=n_{k}$ and we know from (4.21) that $n_{k} \geq C r_{k}^{2}$.

Since every point in the annulus $\mathcal{A}$ is at most a distance $O\left(\varepsilon r_{k}\right)$ from a point where $\Phi_{k}^{\mathrm{TF}}$ vanishes, the gradient estimate (4.25) implies a bound on the potential in the annulus:

$$
\sup _{x \in \mathcal{A}}\left|\Phi_{k}^{\mathrm{TF}}(x)\right| \leq C \varepsilon r_{k} \frac{n_{k}}{\delta \cdot r_{k}} \leq C \frac{\varepsilon}{\delta} r_{k}^{2}(1+o(1))
$$

where in the second inequality we used that $n_{k}$ is in any case smaller than $4 r_{k}^{2}(1+o(1))$ for large $k$ by Lemma 4.4. With (4.28) at our disposal, we can conclude in two different ways:

First proof. By Eq. (3.8) the bound (4.28) implies a bound on the largest radius $\bar{r}_{k}$ of a disk $D\left(0, \bar{r}_{k}\right)$ containing the support of $\sigma_{k}^{\mathrm{TF}}$ :

$$
\bar{r}_{k} \leq\left((1+\delta) r_{k}+C r_{k} \sqrt{\frac{\varepsilon}{\delta}}\right)(1+o(1)) .
$$

Choosing $\delta=\varepsilon^{1 / 3}$ to optimize the above we obtain

$$
\bar{r}_{k} \leq r_{k}\left(1+C \varepsilon^{1 / 3}\right)(1+o(1))
$$

Since $0 \leq \sigma_{k}^{\mathrm{TF}} \leq 1$ this implies

$$
n_{k}=\int_{\mathbb{R}^{2}} \sigma_{k}^{\mathrm{TF}} \leq \pi \bar{r}_{k}^{2} \leq \pi r_{k}^{2}\left(1+C \varepsilon^{1 / 3}\right)^{2}(1+o(1))
$$

and thus, since $\varepsilon>0$ is arbitrary,

$$
\varrho_{\max }=\lim _{k \rightarrow \infty} \frac{n_{k}}{\pi r_{k}^{2}} \leq 1
$$

Second proof. Let $\bar{\Phi}$ be the circular average (3.40) of $\Phi_{k}^{\mathrm{TF}}$. By Newton's theorem [41, Theorem 9.7] we have in the complement of $D\left(0, r_{k}\right)$

$$
\partial_{r} \bar{\Phi}=\frac{M(r)-n_{k}}{r} \text { with } M(r)=\int_{D(0, r)} \sigma^{\mathrm{TF}} .
$$

Thus, picking some fixed $\delta^{\prime}>\delta>0$,

$$
\bar{\Phi}\left(\left(1+\delta^{\prime}\right) r_{k}\right)-\bar{\Phi}\left((1+\delta) r_{k}\right) \leq\left(\pi r_{k}^{2}\left(1+\delta^{\prime}\right)^{2}-n_{k}\right) \log \frac{1+\delta^{\prime}}{1+\delta}
$$


because $M(r) \leq \pi r^{2}$ is an increasing function. By construction we have, for large $k$, $n_{k} \geq \pi r_{k}^{2} \varrho_{\max }(1+o(1))$ and so

$$
\bar{\Phi}\left(\left(1+\delta^{\prime}\right) r_{k}\right)-\bar{\Phi}\left((1+\delta) r_{k}\right) \leq \pi r_{k}^{2}\left(\left(1+\delta^{\prime}\right)^{2}-\varrho_{\max }-o(1)\right) \log \frac{1+\delta^{\prime}}{1+\delta} .
$$

Combining with (4.28) we deduce

$$
\pi r_{k}^{2}\left(\left(1+\delta^{\prime}\right)^{2}-\varrho_{\max }-o(1)\right) \log \frac{1+\delta^{\prime}}{1+\delta} \geq-C \frac{\varepsilon}{\delta} r_{k}^{2}
$$

and thus

$$
\varrho_{\max } \leq\left(1+\delta^{\prime}\right)^{2}(1+o(1))+C\left(\log \frac{1+\delta^{\prime}}{1+\delta}\right)^{-1} \frac{\varepsilon}{\delta}
$$

for any $\varepsilon>0$ and $\delta^{\prime}>\delta>0$ where the $o(1)$ goes to zero when $k \rightarrow \infty$. We may thus take the limits $k \rightarrow \infty, \varepsilon \rightarrow 0, \delta \rightarrow 0, \delta^{\prime} \rightarrow 0$, in this order to deduce that $\varrho_{\max } \leq 1$ as desired.

4.3. Applications. After scaling, $x \rightarrow z=\sqrt{\pi \ell} x$, Theorem 4.3 applies to the Hamiltonian

$$
H_{N}\left(Z_{N}\right)=\sum_{j=1}^{N}\left|z_{j}\right|^{2}-2 \ell \sum_{1 \leq i<j \leq N} \log \left|z_{i}-z_{j}\right|+W\left(Z_{N}\right)
$$

for any superharmonic function $W$ of the $N$ variables. To obtain a corresponding result for low-temperature Gibbs states, we shall later use a Feynman-Hellmann argument and thus need to obtain bounds for a perturbed version of (4.33):

$$
H_{N}^{\varepsilon}\left(Z_{N}\right)=H_{N}\left(Z_{N}\right)+\varepsilon \sum_{i=1}^{N} U\left(z_{i}\right)
$$

where $\varepsilon>0$ is a small enough number.

Proposition 4.6 (Density bound for perturbed plasma ground states). Assume $U \in$ $C^{2}\left(\mathbb{R}^{2}\right)$ with $\Delta U$ uniformly bounded on $\mathbb{R}^{2}$. Let $Z_{N}^{0}=\left(z_{1}^{0}, \ldots, z_{N}^{0}\right)$ be a minimizing configuration for (4.34) and

$$
\mu_{\varepsilon}^{0}(z)=\frac{1}{N} \sum_{i=1}^{N} \delta\left(z-z_{i}^{0}\right)
$$

the corresponding empirical measure. For any open set with Lipschitz boundary $\Omega$, denote by $\Omega_{r}$ the set obtained by dilating $\Omega$ around some origin by a factor $r \underset{N \rightarrow \infty}{\longrightarrow} \infty$. Then, as $N \rightarrow \infty$

$$
\int_{\Omega_{r}} \mu_{\varepsilon}^{0} \leq \frac{1}{N \ell \pi}\left|\Omega_{r}\right|\left(1+\frac{\varepsilon}{4}\|\Delta U\|_{\infty}\right)(1+o(1))
$$

with $\|\Delta U\|_{\infty}=\sup _{\mathbb{R}^{2}}|\Delta U|$. 
Proof. For $\varepsilon=0$ and $\Omega$ a disk this is just a combination of Proposition 4.2 and Theorem 4.3, together with the scaling $x \rightarrow \sqrt{\pi \ell} z$. For the perturbed Hamiltonian, we add and subtract

$$
\frac{\varepsilon}{4} \sum_{i=1}^{N}\|\Delta U\|_{\infty}\left|z_{i}\right|^{2}
$$

and use the fact that $U(z)-\frac{1}{4}\|\Delta U\|_{\infty}|z|^{2}$ is superharmonic. Hence

$$
\varepsilon \sum_{i=1}^{N}\left(U\left(z_{i}\right)-\frac{1}{4}\|\Delta U\|_{\infty}\left|z_{i}\right|^{2}\right)
$$

can be absorbed in $W$ and we only need to reproduce the proof by writing (4.34) in the manner

$$
H_{N}^{\varepsilon}\left(Z_{N}\right)=\left(1+\frac{\varepsilon}{4}\|\Delta U\|_{\infty}\right)\left[\sum_{j=1}^{N}\left|z_{j}\right|^{2}-2 \tilde{\ell} \sum_{1 \leq i<j \leq N} \log \left|z_{i}-z_{j}\right|+\tilde{W}\left(Z_{N}\right)\right] .
$$

where $\tilde{\ell}=\left(1+\frac{\varepsilon}{4}\|\Delta U\|_{\infty}\right)^{-1} \ell$ and $\widetilde{W}$ is again superharmonic in each variable.

To obtain the result for a general domain $\Omega_{r}$, we argue by covering it with disks, as in the "Cheese Theorem" [43, Section 14.4] which quantifies how effectively a region can be approximately covered with non-overlapping disks. We tile $\Omega_{r}$ with disks of radius $\sqrt{r}$, leaving an open set of area at most $O(r)$ untiled. In the disks, we argue as previously. In the remaining untiled area we can use the rougher bound on the minimal separation of points, Lemma 4.4 to show that it contains at most $O(r)$ points. This is of smaller order than the main contribution to (4.36), which comes from the disks, whose union contains $O\left(r^{2}\right)$ points, and the result follows.

\section{Local Density Bounds for Classical Gibbs States}

Here we prove our main result Theorem 2.1. We proceed in two steps which are roughly as follows:

- we first obtain the result for fully-correlated states having angular momentum bounded by $C N^{2}$ for some $C>0$. These have the bulk of their density contained in a region that is not vastly larger than the extension of the Laughlin state.

- next we eliminate the a priori angular momentum bound by a localization procedure.

5.1. Conditional local density bound. In order to combine neatly with the localization procedure used in Sect. 5.2 below, we must work in a slightly more general setting than discussed in Sect. 2.3. Let $\boldsymbol{\mu}$ be a $N$-body probability density that we can write in the form

$$
\boldsymbol{\mu}\left(z_{1}, \ldots, z_{N}\right)=\left|\Psi_{\mathrm{Lau}}\left(z_{1}, \ldots, z_{N}\right)\right|^{2} \prod_{j=1}^{N} \exp \left(-\frac{\left|z_{j}-a\right|^{2}}{L^{2}}\right) G\left(z_{1}, \ldots, z_{N}\right)
$$


where $L>0$ is a number and $\log G$ is subharmonic (see e.g. [41, Chapter 9] for equivalent characterizations):

$$
G \geq 0 \text { and } \Delta_{j} \log G \geq 0 \text { for any } j=1 \ldots N .
$$

Note that $G$ need not be the modulus of an analytic function here. It could, in fact, be of the form $G=\left|F_{1}\right|+\left|F_{2}\right|$ with $F_{1}$ and $F_{2}$ analytic, and we shall use this later, see Lemma 5.2 below.

Marginal probabilities of $\boldsymbol{\mu}$ will be denoted as

$$
\boldsymbol{\mu}^{(k)}\left(z_{1}, \ldots, z_{k}\right)=\int_{\mathbb{R}^{2(N-k)}} \boldsymbol{\mu}\left(z_{1}, \ldots, z_{N}\right) d z_{k+1} \ldots d z_{N} .
$$

In this subsection we prove the following on the first marginal density:

Theorem 5.1 (Conditional density bound). Take $L \geq 1$ in (5.1). For any disk $D$ of radius $r=N^{\alpha}, \alpha>1 / 4$, any choice of origin $a \in \mathbb{R}^{2}$

$$
\int_{D} \boldsymbol{\mu}^{(1)} \leq \frac{1}{N \ell \pi}|D|\left(1+o_{D}(1)+O\left(L^{-2}\right)\right)+C N^{-1-2 \alpha^{\prime}} \int_{\mathbb{R}^{2}}|z-a|^{2} \boldsymbol{\mu}^{(1)}(z) d z
$$

for any $\alpha^{\prime}<\alpha$ where $|D|$ is the area of the disk and $o_{D}(1)$ tends to zero as $|D| \rightarrow \infty$.

To see how this relates to our main result, think of the case where $\mu=\left|\Psi_{F}\right|^{2}$ is the density of a fully-correlated state, that is where $L=+\infty$ and $G=|F|$ with $F$ analytic. Assuming a total angular momentum of order $N^{2}$

$$
\left\langle\Psi_{F}\left|\sum_{j=1}^{N} z_{j} \partial_{z_{j}}-\bar{z}_{j} \partial_{\bar{z}_{j}}\right| \Psi_{F}\right\rangle \leq C N^{2}
$$

we have (see e.g. [58, Equation (2.18)])

$$
\int_{\mathbb{R}^{2}}|z|^{2} \boldsymbol{\mu}^{(1)}(z) d z \leq C N .
$$

Theorem 2.1 follows in this case by taking $a=0$ : the main (first) term behaves as $N^{-1+2 \alpha} \gg N^{-1+2 \alpha^{\prime}}$ and the error (second term) as $N^{-2 \alpha^{\prime}}$, thus the statement is meaningful for $\alpha>1 / 4$.

Proof. The method is basically the same as in [60, Section 3.1]. We first write $\boldsymbol{\mu}$ as a Boltzmann-Gibbs factor:

$$
\begin{aligned}
\boldsymbol{\mu}\left(z_{1}, \ldots, z_{N}\right)= & \frac{1}{\mathcal{Z}_{N}} \exp \left(-\mathbb{H}_{N}\left(z_{1}, \ldots, z_{N}\right)\right) \\
\mathbb{H}_{N}\left(Z_{N}\right)= & \sum_{j=1}^{N}\left(\left|z_{j}\right|^{2}+\frac{\left|z_{j}-a\right|^{2}}{L^{2}}\right) \\
& -2 \ell \sum_{1 \leq i<j \leq N} \log \left|z_{i}-z_{j}\right|-\log G\left(z_{1}, \ldots, z_{N}\right)
\end{aligned}
$$


with $\mathcal{Z}_{N}$ a normalization constant. Thus $\boldsymbol{\mu}$ minimizes the free-energy functional

$$
\mathcal{E}_{N}^{0}[\boldsymbol{v}]:=\int_{\mathbb{R}^{2 N}} \mathbb{H}_{N}\left(Z_{N}\right) d \boldsymbol{v}\left(Z_{N}\right)+\int_{\mathbb{R}^{2 N}} \boldsymbol{v} \log \boldsymbol{v}
$$

over all $N$-particles probability densities $\boldsymbol{v}$. We denote by $E_{N}^{0}=-\log \mathcal{Z}_{N}$ the minimum value.

For $r, \delta>0$ let $U_{r}$ denote the negative characteristic function of a disk with radius $r$. Let $U_{r, \delta}$ be a regularization over a distance $\delta$ so that

$$
\left\|\Delta U_{r, \delta}\right\|_{\infty} \leq C \delta^{-2}
$$

We may choose the regularization in such a way that

$$
U_{r+\delta} \leq U_{r, \delta} \leq U_{r}
$$

Consider now a perturbed version of the above classical Hamiltonian $\mathbb{H}_{N}$ :

$$
\mathbb{H}_{N}^{\varepsilon}\left(Z_{N}\right)=\mathbb{H}_{N}\left(Z_{N}\right)+\varepsilon \sum_{j=1}^{N} U_{r, \delta}\left(z_{j}\right)
$$

along with the associated free-energy functional

$$
\mathcal{E}_{N}^{\varepsilon}[\boldsymbol{v}]:=\int_{\mathbb{R}^{2 N}} \mathbb{H}_{N}^{\varepsilon}\left(Z_{N}\right) d \boldsymbol{v}\left(Z_{N}\right)+\int_{\mathbb{R}^{2 N}} \boldsymbol{v} \log \boldsymbol{v}
$$

whose minimum we denote by $E_{N}^{\varepsilon}$. By definition we have

$$
\mathcal{E}_{N}^{\varepsilon}[\boldsymbol{\mu}]=E_{N}^{0}+\varepsilon N \int_{\mathbb{R}^{2}} U_{r, \delta} \boldsymbol{\mu}^{(1)}
$$

and we will obtain our density estimate from upper and lower bounds to this free energy. Free-energy upper bound. We need an upper bound on $E_{N}^{0}$. We use the trial state

$$
\boldsymbol{\mu}^{\mathrm{t}}\left(z_{1}, \ldots, z_{N}\right):=\left(\frac{1}{\pi \eta^{2}}\right)^{N} \mathbb{1}_{z_{1} \in D\left(z_{1}^{0}, \eta\right)} \otimes \cdots \otimes \mathbb{1}_{z_{N} \in D\left(z_{N}^{0}, \eta\right)} .
$$

where $Z_{N}^{0}=\left(z_{1}^{0}, \ldots, z_{N}^{0}\right)$ is a minimizing configuration for $\mathbb{H}_{N}$. We have

$$
\int_{\mathbb{R}^{2 N}} \boldsymbol{\mu}^{\mathrm{t}} \log \boldsymbol{\mu}^{\mathrm{t}}=-N \log \left(\pi \eta^{2}\right)
$$

and

$$
\begin{aligned}
\int_{\mathbb{R}^{2 N}} & \mathbb{H}_{N}\left(Z_{N}\right) \boldsymbol{\mu}^{\mathrm{t}}\left(Z_{N}\right) d Z_{N} \\
\leq & \sum_{j=1}^{N}\left(\frac{1}{\pi \eta^{2}} \int_{D\left(z_{j}^{0}, \eta\right)}|z|^{2} d z+\frac{1}{L^{2} \pi \eta^{2}} \int_{D\left(z_{j}^{0}, \eta\right)}\left|z_{j}-a\right|^{2} d z\right) \\
& -2 \ell \sum_{1 \leq i<j \leq N} \log \left|z_{i}^{0}-z_{j}^{0}\right|-\log G\left(Z_{N}^{0}\right),
\end{aligned}
$$


because superharmonicity in each variable of the function

$$
\left(z_{1}, \ldots, z_{N}\right) \mapsto-2 \ell \sum_{1 \leq i<j \leq N} \log \left|z_{i}-z_{j}\right|-\log G\left(Z_{N}\right)
$$

implies that it cannot increase upon taking an average over disks centered at the $z_{i}^{0}$ 's (see e.g. [41, Chapter 9]). For the one-body term we have, integrating in polar coordinates

$$
\frac{1}{\pi \eta^{2}} \int_{D\left(z_{j}^{0}, \eta\right)}|z|^{2} d z=\frac{1}{\pi \eta^{2}} \int_{D(0, \eta)}\left|z+z_{j}^{0}\right|^{2} d z=\left|z_{j}^{0}\right|^{2}+\frac{1}{\pi \eta^{2}} \int_{D(0, \eta)}|z|^{2} d z=\left|z_{j}^{0}\right|^{2}+\frac{\eta^{2}}{2}
$$

and by the same token, since $L \geq 1$

$$
\frac{1}{L^{2} \pi \eta^{2}} \int_{D\left(z_{j}^{0}, \eta\right)}\left|z_{j}-a\right|^{2} d z \leq \frac{1}{L^{2}}\left|z_{j}^{0}-a\right|^{2}+\frac{\eta^{2}}{2} .
$$

Fixing $\eta$ we thus deduce

$$
\mathcal{E}_{N}^{\varepsilon}[\boldsymbol{\mu}] \leq \min _{\mathbb{R}^{2 N}} \mathbb{H}_{N}^{0}+\varepsilon N \int_{\mathbb{R}^{2}} U_{r, \delta} \boldsymbol{\mu}^{(1)}+C N
$$

Free-energy lower bound. By positivity of the relative entropy (Jensen's inequality) we have, for any pair of probability measures $\boldsymbol{v}, \boldsymbol{v}^{\mathrm{t}}$,

$$
\int_{\mathbb{R}^{2 N}} \boldsymbol{v} \log \boldsymbol{v} \geq \int_{\mathbb{R}^{2 N}} \boldsymbol{v} \log \boldsymbol{v}^{\mathrm{t}}
$$

Taking

$$
v^{\mathrm{t}}\left(z_{1}, \ldots, z_{N}\right):=(\pi N)^{-N} \prod_{j=1}^{N} \exp \left(-\frac{\left|z_{j}-a\right|^{2}}{N}\right)
$$

we deduce that for any probability measure

$$
\int_{\mathbb{R}^{2 N}} \boldsymbol{v} \log \boldsymbol{v} \geq-\int_{\mathbb{R}^{2}}|z-a|^{2} \boldsymbol{v}^{(1)}(z) d z-O(N \log N) .
$$

Thus

$$
\begin{aligned}
\mathcal{E}_{N}^{\varepsilon}[\boldsymbol{\mu}] & \geq \int_{\mathbb{R}^{2 N}} \mathbb{H}_{N}^{\varepsilon}\left(Z_{N}\right) \boldsymbol{\mu}\left(Z_{N}\right) d Z_{N}-\int_{\mathbb{R}^{2}}|z-a|^{2} \boldsymbol{\mu}^{(1)}(z) d z-O(N \log N) \\
& \geq \min _{\mathbb{R}^{2 N}} \mathbb{H}_{N}^{\varepsilon}-\int_{\mathbb{R}^{2}}|z-a|^{2} \boldsymbol{\mu}^{(1)}(z) d z-O(N \log N) \\
& \geq \min _{\mathbb{R}^{2 N}} \mathbb{H}_{N}^{0}+\varepsilon N \int_{\mathbb{R}^{2}} U_{r, \delta} \mu_{\varepsilon}^{0}-\int_{\mathbb{R}^{2}}|z-a|^{2} \boldsymbol{\mu}^{(1)}(z) d z-O(N \log N)
\end{aligned}
$$

where $\mu_{\varepsilon}^{0}$ is the empirical measure of a minimizing configuration for $\mathbb{H}_{N}^{\varepsilon}$.

Conclusion. Combining with (5.20) we deduce

$$
\int_{\mathbb{R}^{2}} U_{r, \delta} \boldsymbol{\mu}^{(1)} \geq \int_{\mathbb{R}^{2}} U_{r, \delta} \mu_{\varepsilon}^{0}-(\varepsilon N)^{-1} \int_{\mathbb{R}^{2}}|z-a|^{2} \boldsymbol{\mu}^{(1)}(z) d z-C \varepsilon^{-1} \log N .
$$


But, by (5.10) we have

$$
\int_{D_{r}} \boldsymbol{\mu}^{(1)}=-\int_{\mathbb{R}^{2}} U_{r} \boldsymbol{\mu}^{(1)} \leq-\int_{\mathbb{R}^{2}} U_{r, \delta} \boldsymbol{\mu}^{(1)}
$$

and

$$
-\int_{\mathbb{R}^{2}} U_{r, \delta} \mu_{\varepsilon}^{0} \leq \int_{D_{r+\delta}} \mu_{\varepsilon}^{0} .
$$

Proposition 4.6 applies to $\mu_{0}^{\varepsilon}$ because it is the empirical measure of a minimizing configuration for (5.7) (compare with (4.34)). All in all we thus have

$$
\begin{aligned}
\int_{D_{r}} \boldsymbol{\mu}^{(1)} \leq & \pi N^{-1}(r+\delta)^{2}\left(1+o(1)+\frac{C}{L^{2}}+\varepsilon \delta^{-2}\right) \\
& +\varepsilon^{-1} \log N+(\varepsilon N)^{-1} \int_{\mathbb{R}^{2}}|z-a|^{2} \boldsymbol{\mu}^{(1)}(z) d z
\end{aligned}
$$

where we have used (5.9) and assumed that $r+\delta \gg 1$. Consider now the case that $r=N^{\alpha}$ with $\alpha>1 / 4$. We can then clearly choose $\varepsilon, \delta$ depending on $r$ so as to have

$$
r \gg \delta \gg \varepsilon^{1 / 2} \gg(N \log N)^{1 / 2} r^{-1},
$$

which implies

$$
N^{-1} r^{2} \gg \varepsilon^{-1} \log N \text { and } 1 \gg \varepsilon \delta^{-2}
$$

and thus (5.4) follows.

5.2. Localization procedure. We now want to extend Theorem 5.1 by dropping the angular momentum constraint. We use a rather standard localization procedure, see e.g. $[38,54]$ for its discussion in more general contexts. In order for the localization method to combine efficiently with what we have proved so far, we need the following elementary lemma:

Lemma 5.2 (The PL class). The class of functions $G$ satisfying (5.2) is closed under addition. Thus any function $G$ that can be written in the manner $G=\sum_{j} \alpha_{j}\left|F_{j}\right|$ with $\alpha_{j}$ positive numbers and $F_{j}$ analytic functions satisfies (5.2).

Proof. This is a well-known fact, see e.g. [52, Paragraphs 2.12 to 2.14] where (5.2) is called the PL class. One can verify it by a simple computation: if $G, H$ satisfy (5.2) we have

$$
\Delta \log G=\frac{G \Delta G-|\nabla G|^{2}}{G^{2}} \geq 0
$$

and the same for $H$, thus

$$
\begin{aligned}
(G+H)^{2} \Delta \log (G+H)= & (G+H)(\Delta G+\Delta H)-|\nabla G+\nabla H|^{2} \\
= & G \Delta G-\left|\nabla G^{2}\right|+H \Delta H-|\nabla H|^{2}+G \Delta H \\
& +H \Delta G-2 \nabla G \cdot \nabla H \\
\geq & \frac{G}{H}|\nabla H|^{2}+\frac{H}{G}|\nabla G|^{2}-2 \nabla G \cdot \nabla H \\
\geq & \left|\sqrt{\frac{G}{H}} \nabla H-\sqrt{\frac{H}{G}} \nabla G\right|^{2} \geq 0 .
\end{aligned}
$$


The rest of the statement follows because any $|F|$ with $F$ analytic satisfies (5.2) as remarked after Eq. (2.20). See also [30, Page 84].

We now proceed to extending Theorem 5.1 to states not necessarily satisfying (5.5). This will conclude the

Proof of Theorem 2.1. For $\Psi_{F} \in \mathcal{L}_{\ell}^{N}$ define

$$
\boldsymbol{\mu}_{F}\left(z_{1}, \ldots, z_{N}\right)=\left|\Psi_{F}\left(z_{1}, \ldots, z_{N}\right)\right|^{2} .
$$

Localization procedure. We define a smooth partition of unity on $\mathbb{R}^{2}$

$$
\chi^{2}+\eta^{2} \equiv 1, \quad \chi^{2}(z)=e^{-\frac{|z-a|^{2}}{L^{2}}}
$$

with $L \rightarrow \infty$ a length scale to be optimized over. Consider the classical $M$-particle state $^{6} \boldsymbol{\mu}_{M}$ built from $\boldsymbol{\mu}$ by localizing exactly $M$ particles and integrating out the other:

$$
\boldsymbol{\mu}_{M}\left(z_{1}, \ldots, z_{M}\right):=\prod_{j=1}^{M} \chi^{2}\left(z_{j}\right) \int_{\mathbb{R}^{2(N-M)}} \prod_{j=M+1}^{N} \eta^{2}\left(z_{j}\right) \mu_{F}\left(Z_{N}\right) d z_{M+1} \ldots d z_{N} .
$$

Observe that, using

$$
\prod_{j=1}^{N}\left(\chi^{2}\left(z_{j}\right)+\eta^{2}\left(z_{j}\right)\right)=1
$$

and symmetry under particle exchange,

$$
\boldsymbol{\mu}_{F}\left(Z_{N}\right)=\sum_{M=0}^{N}\left(\begin{array}{l}
N \\
M
\end{array}\right) \prod_{j=1}^{M} \chi^{2}\left(z_{j}\right) \prod_{j=M+1}^{N} \eta^{2}\left(z_{j}\right) \mu_{F}\left(Z_{N}\right)
$$

and thus the $L^{2}$-normalization of $\Psi_{F}$ implies

$$
\sum_{M=0}^{N}\left(\begin{array}{l}
N \\
M
\end{array}\right) \int_{\mathbb{R}^{2 M}} \boldsymbol{\mu}_{M}=1
$$

Next, recalling (2.3) and (5.3) we denote by $\boldsymbol{\mu}_{F}^{(1)}=N^{-1} \rho_{F}$ the one-particle probability density of $\Psi_{F}$. By symmetry under particle exchange again, we have

$$
\begin{aligned}
& \chi^{2}\left(z_{1}\right) \boldsymbol{\mu}_{F}^{(1)}\left(z_{1}\right) \\
& \quad=\int_{\mathbb{R}^{2(N-1)}} \chi^{2}\left(z_{1}\right) \boldsymbol{\mu}_{F}\left(Z_{N}\right) d z_{2} \ldots d z_{N} \\
& \quad=\int_{\mathbb{R}^{2(N-1)}} \chi^{2}\left(z_{1}\right) \prod_{M=2}^{N}\left(\chi^{2}\left(z_{M}\right)+\eta^{2}\left(z_{M}\right)\right) \boldsymbol{\mu}_{F}\left(Z_{N}\right) d z_{2} \ldots d z_{N} \\
& \quad=\sum_{M=1}^{N}\left(\begin{array}{l}
N-1 \\
M-1
\end{array}\right) \int_{\mathbb{R}^{2(N-1)}} \chi^{2}\left(z_{1}\right) \ldots \chi^{2}\left(z_{M}\right) \eta^{2}\left(z_{M+1}\right) \ldots \eta^{2}\left(z_{N}\right) \boldsymbol{\mu}_{F}\left(Z_{N}\right) d z_{2} \ldots d z_{N} \\
& \quad=\sum_{M=1}^{N}\left(\begin{array}{l}
N-1 \\
M-1
\end{array}\right) \boldsymbol{\mu}_{M}^{(1)}\left(z_{1}\right) .
\end{aligned}
$$

\footnotetext{
6 Strictly speaking it is not normalized, thus not a state.
} 
Finally, since clearly $|z-a|^{2} \chi^{2}(z) \leq L^{2+\delta} \chi^{2}(z)$ for arbitrary $\delta>0$ we have

$$
\begin{aligned}
& \sum_{M=1}^{N}\left(\begin{array}{l}
N-1 \\
M-1
\end{array}\right) \int_{\mathbb{R}^{2}}\left|z_{1}-a\right|^{2} \boldsymbol{\mu}_{M}^{(1)}\left(z_{1}\right) d z_{1} \\
& \quad=\sum_{M=1}^{N}\left(\begin{array}{l}
N-1 \\
M-1
\end{array}\right) \int_{\mathbb{R}^{2 M}}\left|z_{1}-a\right|^{2} \prod_{j=1}^{M} \chi^{2}\left(z_{j}\right) \prod_{j=M+1}^{N} \eta^{2}\left(z_{j}\right) \boldsymbol{\mu}_{F}\left(Z_{N}\right) d z_{1} \ldots d z_{N} \\
& \quad \leq L^{2+\delta} \int_{\mathbb{R}^{2 M}} \sum_{M=1}^{N}\left(\begin{array}{l}
N-1 \\
M-1
\end{array}\right) \prod_{j=2}^{M} \chi^{2}\left(z_{j}\right) \prod_{j=M+1}^{N} \eta^{2}\left(z_{j}\right) \boldsymbol{\mu}_{F}\left(Z_{N}\right) d z_{1} \ldots d z_{N} \\
& \quad \leq L^{2+\delta} \int_{\mathbb{R}^{2 M}} \prod_{j=2}^{N}\left(\chi^{2}\left(z_{j}\right)+\eta^{2}\left(z_{j}\right)\right) \boldsymbol{\mu}_{F}\left(Z_{N}\right) d z_{1} \ldots d z_{N}=L^{2+\delta} .
\end{aligned}
$$

Application. Pick a disk $D$ of center $a \in \mathbb{R}^{2}$ and radius $r=N^{\alpha}$ with $\alpha>1 / 4$ and choose

$$
\chi^{2}(z)=\exp \left(-\frac{|z-a|^{2}}{L^{2}}\right)
$$

with $L=N^{\beta}, \beta>\alpha$. Then clearly

$$
\int_{D} \boldsymbol{\mu}_{F}^{(1)} \leq(1+o(1)) \int_{D} \chi^{2} \boldsymbol{\mu}_{F}^{(1)}
$$

and we expect the right-hand side to be at most of order $N^{-1+2 \alpha}$. To estimate it, we start from (5.40) and first brutally get rid of terms with small $M$ for they have too few particles to contribute to the density at this scale:

$$
\begin{aligned}
\int_{D} \chi^{2} \boldsymbol{\mu}_{F}^{(1)} & =\sum_{M=1}^{N}\left(\begin{array}{l}
N \\
M
\end{array}\right) \frac{M}{N} \int_{D} \boldsymbol{\mu}_{M}^{(1)} \\
& \leq N^{\gamma-1} \sum_{M=1}^{N^{\gamma}}\left(\begin{array}{l}
N \\
M
\end{array}\right) \int_{D} \boldsymbol{\mu}_{M}^{(1)}+\sum_{M=N^{\gamma}}^{N}\left(\begin{array}{l}
N \\
M
\end{array}\right) \frac{M}{N} \int_{D} \boldsymbol{\mu}_{M}^{(1)} \\
& \leq N^{\gamma-1} \sum_{M=1}^{N^{\gamma}}\left(\begin{array}{l}
N \\
M
\end{array}\right) \int_{\mathbb{R}^{2 M}} \boldsymbol{\mu}_{M}+\sum_{M=N^{\gamma}}^{N}\left(\begin{array}{l}
N \\
M
\end{array}\right) \frac{M}{N} \int_{D} \boldsymbol{\mu}_{M}^{(1)} \\
& \leq N^{\gamma-1}+\sum_{M=N^{\gamma}}^{N}\left(\begin{array}{l}
N \\
M
\end{array}\right) \frac{M}{N} \int_{D} \boldsymbol{\mu}_{M}^{(1)}
\end{aligned}
$$

where we used that

$$
\left(\begin{array}{l}
N \\
M
\end{array}\right)=\frac{N}{M}\left(\begin{array}{l}
N-1 \\
M-1
\end{array}\right)
$$

and inserted (5.39). We pick $\gamma<2 \alpha$ to render the first term $N^{\gamma-1}$ in the above much smaller than the expected $N^{-1+2 \alpha}$, so that we may focus on the second one. 
We thus need to bound the 1-particle probability density $\boldsymbol{\mu}_{M}^{(1)}$, first marginal of the $M$-particles state $\boldsymbol{\mu}_{M}$. Recalling (5.37) we can write

$$
\mu_{M}\left(z_{1}, \ldots, z_{k}\right)=\left|\Psi_{\text {Lau }}\left(z_{1}, \ldots, z_{M}\right)\right|^{2} \exp \left(-\sum_{j=1}^{M} \frac{\left|z_{j}-a\right|^{2}}{L^{2}}\right) G_{M}\left(z_{1}, \ldots, z_{M}\right)
$$

where ( $c_{M}$ is a positive constant ensuring normalization of (5.46))

$$
\begin{aligned}
G_{M}\left(z_{1}, \ldots, z_{M}\right)= & c_{M} \int_{\mathbb{R}^{2(N-M)}} \prod_{j=M+1}^{N} \eta^{2}\left(z_{j}\right) e^{-\left|z_{j}\right|^{2}} \prod_{M+1 \leq i<j \leq N}\left|z_{i}-z_{j}\right|^{2 \ell} \\
& \left|F\left(z_{1}, \ldots, z_{N}\right)\right|^{2} \prod_{i=1}^{M} \prod_{j=M+1}^{N}\left|z_{i}-z_{j}\right|^{2 \ell} d z_{M+1} \ldots d z_{N} .
\end{aligned}
$$

At fixed $z_{M+1}, \ldots, z_{N}$, the integrand in the above is the squared modulus of an analytic function of the variables $z_{1}, \ldots, z_{M}$ (they appear only in the second line) and is thus subharmonic. Since the PL class is closed under addition, c.f. Lemma 5.2, we deduce that $G_{M}$ satisfies Assumption (5.2). We can thus apply (5.4) to the normalized version of $\boldsymbol{\mu}_{M}$ and deduce

$$
\begin{aligned}
\frac{\int_{D} \mu_{M}^{(1)}}{\int_{\mathbb{R}^{2 M} \mu_{M}} \leq} & \frac{1}{M \ell \pi}|D|\left(1+o_{D}(1)+O\left(L^{-2}\right)\right) \\
& +C M^{-1-2 \alpha^{\prime}}\left(\int_{\mathbb{R}^{2 M}} \mu_{M}\right)^{-1} \int_{\mathbb{R}^{2}}|z-a|^{2} \mu_{M}^{(1)}(z) d z
\end{aligned}
$$

for any $\alpha^{\prime}<\alpha$. Next, we sum the above inequalities from $M=N^{\gamma}$ to $N$ and insert the result in (5.44) to obtain

$$
\begin{aligned}
\int_{D} \chi^{2} \boldsymbol{\mu}_{F}^{(1)} \leq & \sum_{M=N^{\gamma}}^{N}\left(\begin{array}{l}
N \\
M
\end{array}\right) \frac{M}{N}\left(\int_{\mathbb{R}^{2 M}} \boldsymbol{\mu}_{M}\right) \frac{|D|}{\pi \ell M}\left(1+o_{N}(1)\right) \\
& +C N^{-\gamma-2 \gamma \alpha^{\prime}} \sum_{M=N \gamma}^{N}\left(\begin{array}{l}
N \\
M
\end{array}\right) \frac{M}{N} \int_{\mathbb{R}^{2}}|z-a|^{2} \boldsymbol{\mu}_{M}^{(1)}(z) d z+N^{\gamma-1}
\end{aligned}
$$

where we have used that we sum only terms where $M \gg 1$ to ensure that the $o_{D}(1)$ terms are indeed small. We next insert (5.39) in the first sum and use (5.45) in combination with (5.41) to deal with the second one, obtaining

$$
\int_{D} \chi^{2} \mu_{F}^{(1)} \leq \frac{|D|}{\pi \ell N}\left(1+o_{N}(1)\right)+C N^{-\gamma-2 \gamma \alpha^{\prime}+(2+\delta) \beta}+N^{\gamma-1} .
$$

To optimize over $\gamma$ we take

$$
\gamma=\frac{1}{2+2 \alpha^{\prime}}+\frac{(1+\delta) \beta}{2+2 \alpha^{\prime}}
$$


and since we are at liberty to choose $\alpha^{\prime}, \beta$ arbitrarily close to $\alpha$ and $\delta$ arbitrarily small, we can render $\gamma$ arbitrarily close to $1 / 2$, so that the error terms in (5.50) become $O\left(N^{-1 / 2+\varepsilon}\right)$ for arbitrarily small $\varepsilon>0$. For $\alpha>1 / 4$ we deduce

$$
\int_{D} \boldsymbol{\mu}_{F}^{(1)} \leq(1+o(1)) \int_{D} \chi^{2} \mu_{F}^{(1)} \leq \frac{|D|}{\pi \ell N}\left(1+o_{N}(1)\right)
$$

as desired because the main term is of order $N^{-1+2 \alpha}$. To deduce the general result (2.5) we can simply replace indicative functions of disks by indicative functions of more general open sets everywhere in Sect. 5, relying on (4.36) for a general open set.

\section{Energy in Confining Potentials}

We now provide the

Proof of Corollary 2.3. This is a variation on the arguments of Sect. 5.1, but it is more convenient to work with scaled variables as in [60, Section 3]. We thus write, with the notation of Assumption 2.2

$$
\int_{\mathbb{R}^{2}} V \rho_{F}=N \int_{\mathbb{R}^{2}} U \mu_{F}^{(1)}
$$

with the scaled one-particle probability density

$$
\mu_{F}^{(1)}(x)=\rho_{F}(\sqrt{N} x) .
$$

We will deduce the result from the lower bound

$$
\int_{\mathbb{R}^{2}} U \mu_{F}^{(1)} \geq(1+o(1)) \inf \left\{\int_{\mathbb{R}^{2}} U \mu \mid 0 \leq \mu \leq \frac{1}{\pi \ell}, \int_{\mathbb{R}^{2}} \mu=1\right\}+o(1)
$$

by a simple change of variables.

Let $L>R$ be a large but fixed number, with $R$ as in Assumption 2.2. Define a $U_{L} \leq U$ such that

$$
U_{L}= \begin{cases}U & \text { in } D(0, L) \\ \underline{U} & \text { in } D(0,2 L)^{c}\end{cases}
$$

and $U_{L}$ satisfies the same bounds (2.10)-(2.11) as $\underline{U}$ outside of $D(0, L)$. We then consider (compare with (2.22))

$$
\begin{aligned}
\tilde{H}_{N}^{\varepsilon}\left(z_{1}, \ldots, z_{N}\right)= & \sum_{j=1}^{N}\left(\left|z_{j}\right|^{2}+\varepsilon U_{L}\left(z_{j}\right)\right)-2 \frac{\ell}{N} \sum_{1 \leq i<j \leq N} \log \left|z_{i}-z_{j}\right| \\
& -\frac{2}{N} \log \left|F\left(\sqrt{N} z_{1}, \ldots, \sqrt{N} z_{N}\right)\right|
\end{aligned}
$$

and estimate (recall the notation (2.21))

$$
\tilde{\mathcal{E}}_{N}^{\varepsilon}\left[\mu_{F}\right]:=\int_{\mathbb{R}^{2 N}} \tilde{H}_{N}^{\varepsilon} \mu_{F}+\frac{1}{N} \int_{\mathbb{R}^{2 N}} \mu_{F} \log \mu_{F} .
$$


Since $\mu_{F}$ is the Gibbs state at temperature $1 / N$ associated with $\tilde{H}_{N}^{\varepsilon}$ and $\mu_{F}^{(1)}$ its first marginal we have

$$
\begin{aligned}
\tilde{\mathcal{E}}_{N}^{\varepsilon}\left[\mu_{F}\right]= & \varepsilon N \int_{\mathbb{R}^{2}} U_{L} \mu_{F}^{(1)}+\int_{\mathbb{R}^{2 N}} \tilde{H}_{N}^{0} \mu_{F}+\frac{1}{N} \int_{\mathbb{R}^{2 N}} \mu_{F} \log \mu_{F} \\
& \leq \varepsilon N \int_{\mathbb{R}^{2}} U_{L} \mu_{F}^{(1)}+\min _{\mathbb{R}^{2 N}} \tilde{H}_{N}^{0}+C(\log N+1)
\end{aligned}
$$

using a trial state similar to (5.14) to bound the minimal free energy at temperature $1 / \mathrm{N}$ associated with $\tilde{H}_{N}^{0}$, exactly as in [60, Section 3.1].

On the other hand, by Jensen's inequality

$$
\begin{aligned}
\int_{\mathbb{R}^{2 N}} \mu_{F} \log \mu_{F} & \geq \int_{\mathbb{R}^{2 N}} \mu_{F} \log \left(\frac{e^{-U_{L}}}{\int_{\mathbb{R}^{2}} e^{-U_{L}}}\right)^{\otimes N} \\
& \geq-N \int_{\mathbb{R}^{2}} U_{L} \mu_{F}^{(1)}-C N
\end{aligned}
$$

where we used (2.11) to bound $\int_{\mathbb{R}^{2}} e^{-U_{L}}$. Thus

$$
\begin{aligned}
\tilde{\mathcal{E}}_{N}^{\varepsilon}\left[\mu_{F}\right] & \geq \min _{\mathbb{R}^{2 N}}\left\{\tilde{H}_{N}^{\varepsilon}\left(z_{1}, \ldots, z_{N}\right)-\frac{1}{N} \sum_{j=1}^{N} U_{L}\left(z_{j}\right)\right\}-C \\
& \geq \min _{\mathbb{R}^{2 N}} \tilde{H}_{N}^{0}+(N \varepsilon-1) \int_{\mathbb{R}^{2}} U_{L} \tilde{\mu}_{\varepsilon}^{0}-C
\end{aligned}
$$

where

$$
\tilde{\mu}_{\varepsilon}^{0}(z)=\frac{1}{N} \sum_{i=1}^{N} \delta\left(z-z_{i}^{0}\right)
$$

is the empirical measure of a configuration $\left(z_{1}^{0}, \ldots, z_{N}^{0}\right)$ minimizing the classical Hamiltonian $\tilde{H}_{N}^{\varepsilon}\left(z_{1}, \ldots, z_{N}\right)-\frac{1}{N} \sum_{j=1}^{N} U_{L}\left(z_{j}\right)$. We can apply a (re-scaled version of) Proposition 4.6 to $\tilde{\mu}_{\varepsilon}^{0}$. Thus, for any domain $\Omega_{r}$ obtain from a fixed Lipschitz $\Omega$ by scaling lengths by a factor $r=N^{\alpha^{\prime}}, \alpha^{\prime}>-1 / 2$

$$
\begin{aligned}
\int_{\Omega_{r}} \tilde{\mu}_{\varepsilon}^{0} & \leq \frac{1}{\ell \pi}\left|\Omega_{r}\right|\left(1+\frac{\varepsilon}{4}\left\|\Delta U_{L}\right\|_{\infty}\right)(1+o(1)) \\
& \leq \frac{1}{\ell \pi}\left|\Omega_{r}\right|\left(1+C \varepsilon N^{1-2 \alpha}\right)(1+o(1))
\end{aligned}
$$

where we used (2.9) to estimate $\left\|\Delta U_{L}\right\|_{\infty}$. By a Riemann sum approximation on a grid of side length $N^{\alpha^{\prime}}$ we then have

$$
\int_{\mathbb{R}^{2}} U_{L} \tilde{\mu}_{\varepsilon}^{0} \geq \int_{\mathbb{R}^{2}} U_{L} \hat{\mu}_{\varepsilon}^{0}-C\left\|\nabla U_{L}\right\|_{L^{\infty}} N^{\alpha^{\prime}}
$$

where $\hat{\mu}_{\varepsilon}^{0}$ is a piecewise constant function satisfying

$$
0 \leq \hat{\mu}_{\varepsilon}^{0} \leq \frac{1}{\ell \pi}\left(1+C \varepsilon N^{1-2 \alpha}\right)(1+o(1))
$$


and

$$
\int_{\mathbb{R}^{2}} \hat{\mu}_{\varepsilon}^{0}=1
$$

Since, using (2.9), $\left\|\nabla U_{L}\right\|_{L^{\infty}} \leq C N^{1 / 2-\alpha}$ we deduce

$$
\tilde{\mathcal{E}}_{N}^{\varepsilon}\left[\mu_{F}\right] \geq \min _{\mathbb{R}^{2 N}} \tilde{H}_{N}^{0}+(N \varepsilon-1) \int_{\mathbb{R}^{2}} U_{L} \hat{\mu}_{\varepsilon}^{0}-C N^{1 / 2+\alpha^{\prime}-\alpha}
$$

for any fixed $\alpha>-1 / 2$. Comparing with (6.7) we obtain

$$
\begin{aligned}
\int_{\mathbb{R}^{2}} U \mu_{F}^{(1)} \geq & \left(1-\frac{1}{\varepsilon N}\right) \int_{\mathbb{R}^{2}} U_{L} \hat{\mu}_{\varepsilon}^{0}-\frac{C \log N}{\varepsilon N}-\frac{C N^{1 / 2+\alpha^{\prime}-\alpha}}{\varepsilon N} \\
\geq & \left(1-\frac{1}{\varepsilon N}\right) \inf \left\{\int_{\mathbb{R}^{2}} U_{L} \mu \mid 0 \leq \mu\right. \\
\leq & \left.\frac{1}{\pi \ell}\left(1+C \varepsilon N^{1-2 \alpha}\right)(1+o(1)), \int_{\mathbb{R}^{2}} \mu=1\right\} \\
& -\frac{C \log N}{\varepsilon N}-\frac{C N^{1 / 2+\alpha^{\prime}-\alpha}}{\varepsilon N}
\end{aligned}
$$

where we used that $U \geq U_{L}$. Since $\alpha>0$ and $\alpha^{\prime}$ can be chosen arbitrarily close to $-1 / 2$ in the above, we can choose $\varepsilon=N^{-\delta}$ with some $\delta$ less than, but sufficiently close to, 1 to get

$$
\int_{\mathbb{R}^{2}} U \mu_{F}^{(1)} \geq(1-o(1)) \inf \left\{\int_{\mathbb{R}^{2}} U_{L} \mu \mid 0 \leq \mu \leq \frac{1}{\pi \ell}, \int_{\mathbb{R}^{2}} \mu=1\right\}+o(1)
$$

The continuity of the bathtub energy as a function of the upper density constraint we have just used is a consequence of the explicit solution of the minimization problem [41, Theorem 1.14]. To conclude the proof of (6.3) we note that, under our assumptions on $U$, it suffices to take $L$ large enough but fixed to have

$\inf \left\{\int_{\mathbb{R}^{2}} U_{L} \mu \mid 0 \leq \mu \leq \frac{1}{\pi \ell}, \int_{\mathbb{R}^{2}} \mu=1\right\}=\inf \left\{\int_{\mathbb{R}^{2}} U \mu \mid 0 \leq \mu \leq \frac{1}{\pi \ell}, \int_{\mathbb{R}^{2}} \mu=1\right\}$.

This follows from the fact that minimizers of the bathtub energy are equal to either 0 or $(\pi \ell)^{-1}$ almost everywhere, and that for potentials increasing at infinity, their support is compact.

\section{Conclusions}

By employing a new theorem about multi-particle screening, we have derived sharp local upper bounds on the particle density in ground states of a classical 2D Coulomb gas perturbed by arbitrary many-body potentials which are superharmonic in every variable. We have also derived corresponding bounds for Gibbs states which, via Laughlin's plasma analogy, have direct applications to quantum many-particle states in strong magnetic fields exhibiting the Fractional Quantum Hall Effect (FQHE) and which are fully correlated in order to minimize a repulsive interaction between the particles. Our results hold asymptotically for large particle numbers, on length scales that are small compared 
to the full system's extension. We expect that there is room for improvement as regards the smallest length scale we can afford. This remains an open problem.

The bounds for the classical Coulomb ground states rest on two pillars: First, an "exclusion rule" for ground state configurations which prevents points in such a configuration from entering a screening region defined by the other points in the configuration. Second, a universal density bound that we prove for any configuration of points satisfying the exclusion rule.

For the Gibbs state, the modification of the ground state density caused by the entropy term in the free energy has to be estimated. This is done by perturbing the classical Coulomb Hamiltonian locally and bounding the perturbed free energy, obtaining a density bound via the Feynman-Hellmann principle. This procedure is first carried out under the assumption of an a-priori bound on the angular momentum and then extended to the general case by a localization argument.

Besides the density bounds we also derive lower bounds for the energy of fully correlated FQHE states confined in external potentials. In combination with recently obtained upper bounds [61], this establishes that approximate quantum mechanical ground states in the class of strongly correlated FQHE wave-functions can be obtained by generating uncorrelated quasi-holes on top of the Laughlin state.

Acknowledgements. Open access funding provided by University of Vienna. We received financial support from the French ANR Project ANR-13-JS01-0005-01 (N. Rougerie) and the US NSF Grant PHY-1265118 (E. H. Lieb).

Open Access This article is distributed under the terms of the Creative Commons Attribution 4.0 International License (http://creativecommons.org/licenses/by/4.0/), which permits unrestricted use, distribution, and reproduction in any medium, provided you give appropriate credit to the original author(s) and the source, provide a link to the Creative Commons license, and indicate if changes were made.

\section{References}

1. Almgren, F.J. Jr., Lieb, E.H.: Symmetric decreasing rearrangement is sometimes continuous. J. Am. Math. Soc. 2, 683-773 (1989).

2. Ameur, Y.: A density theorem for weighted Fekete sets. Int. Math. Res. Not. 2017(16) (2017)

3. Ameur, Y.: Repulsion in low temperature $\beta$-ensembles. Commun. Math. Phys. 359, 1079 (2018). arXiv: 1701.04796

4. Ameur, Y., Ortega-Cerdá, J.: Beurling-Landau densities of weighted Fekete sets and correlation kernel estimates. J. Funct. Anal. 263, 1825-1861 (2012)

5. Anderson, G.W., Guionnet, A., Zeitouni, O.: An Introduction to Random Matrices, vol. 118 of Cambridge Studies in Advanced Mathematics. Cambridge University Press, Cambridge (2010)

6. Arovas, S., Schrieffer, J., Wilczek, F.: Fractional statistics and the quantum Hall effect. Phys. Rev. Lett. 53, 722-723 (1984)

7. Bauerschmidt, R., Bourgade, P., Nikula, M., Yau, H.-T.: Local density for two-dimensional one-component plasma (2015). arXiv: 1510.02074

8. Bauerschmidt, R., Bourgade, P., Nikula, M., Yau, H.-T.: The two-dimensional Coulomb plasma: quasi-free approximation and central limit theorem (2016). arXiv: 1609.08582

9. Bergholtz, E.J., Liu, Z.: Topological flat band models and fractional Chern insulators. Int. J. Mod. Phys. B 27, 1330017 (2013)

10. Bétermin, L., Sandier, E.: Renormalized energy and asymptotic expansion of optimal logarithmic energy on the sphere. arXiv e-prints (2014)

11. Bloch, I., Dalibard, J., Zwerger, W.: Many-body physics with ultracold gases. Rev. Mod. Phys. 80, 885964 (2008)

12. Bonacini, M., Knüpfer, H., Röger, M.: Optimal distribution of oppositely charged phases: perfect screening and other properties. SIAM J. Math. Anal. 48, 1128-1154 (2016)

13. Burchard, A., Choksi, R., Topaloglu, I.: Nonlocal shape optimization via interactions of attractive and repulsive potentials. Indiana Univ. J. Math. 67, 375-395 (2018) 
14. Carlen, E.: Some integral identities and inequalities for entire functions and their application to the coherent state transform. J. Funct. Anal. 97, 231-249 (1991)

15. Catto, I., Le Bris, C., Lions, P.-L.: The Mathematical Theory of Thermodynamic Limits: Thomas-Fermi Type Models. Oxford Mathematical Monographs. The Clarendon Press Oxford, University Press, New York (1998)

16. Chafaï, D., Gozlan, N., Zitt, P.-A.: First order asymptotics for confined particles with singular pair repulsions. Ann. Appl. Probab. 24, 2371-2413 (2014)

17. Chafaï, D., Hardy, A., Maïda, M.: Concentration for Coulomb gases and Coulomb transport inequalities (2016). arXiv: 1610.00980

18. Ciftjá, O.: Monte Carlo study of Bose Laughlin wave function for filling factors 1/2,1/4 and 1/6. Europhys. Lett. 74, 486-492 (2006)

19. Ciftjá, O., Wexler, C.: Monte Carlo simulation method for Laughlin-like states in a disk geometry. Phys. Rev. B 67, 075304 (2003)

20. Cooper, N.R.: Rapidly rotating atomic gases. Adv. Phys. 57, 539-616 (2008)

21. de Picciotto, R., Reznikov, M., Heiblum, M., Umansky, V., Bunin, G., Mahalu, D.: Direct observation of a fractional charge. Nature 389, 162-164 (1997)

22. Forrester, P.J.: Log-Gases and Random Matrices, vol. 34 of London Mathematical Society Monographs Series. Princeton University Press, Princeton, NJ (2010)

23. Frank, R.L., Lieb, E.H.: A 'liquid-solid' phase transition in a simple model for swarming, based on the 'no flat-spots' theorem for subharmonic functions. Indiana Univ. Math. J. (2016). arXiv:1607.07971 (in press)

24. Friedman, A.: Variational Principles and Free-Boundary Problems. Robert E. Krieger Publishing Co., Malabar (1988)

25. Girvin, S.: Introduction to the fractional quantum Hall effect. Sémin. Poincaré 2, 54-74 (2004)

26. Haldane, F.D.M.: Fractional quantization of the Hall effect: a hierarchy of incompressible quantum fluid states. Phys. Rev. Lett. 51, 605-608 (1983)

27. Hardy, A.: A note on large deviations for 2D Coulomb gas with weakly confining potential. Electron. Commun. Probab. 17, 1-12 (2012)

28. Hardy, A., Kuijlaars, A.: Weakly admissible vector equilibrium problems. J. Approx. Theory 164, 854$868(2012)$

29. Jain, J.K.: Composite Fermions. Cambridge University Press, Cambridge (2007)

30. Klimek, M.: Pluripotential Theory. Oxford University Press, Oxford (1991)

31. Laughlin, R.B.: Anomalous quantum Hall effect: an incompressible quantum fluid with fractionally charged excitations. Phys. Rev. Lett. 50, 1395-1398 (1983)

32. Laughlin, R.B.: Elementary theory: the incompressible quantum fluid. In: Prange R.E., Girvin S.E. (eds.) The quantum Hall effect. Springer, Heidelberg (1987)

33. Laughlin, R.B.: Nobel lecture: fractional quantization. Rev. Mod. Phys. 71, 863-874 (1999)

34. Leblé, T.: Local microscopic behavior for 2D Coulomb gases. Trans. Probab. Theory Relat. Fields 169, 931 (2017). arXiv:1510.01506

35. Leblé, T., Serfaty, S.: Large deviation principle for empirical fields of Log and Riesz gases (2015). arXiv: 1502.02970

36. Leblé, T., Serfaty, S.: Fluctuations of two-dimensional Coulomb gases. S. Geom. Funct. Anal. 28, 443 (2018). arXiv:1609.08088

37. Leblé, T., Serfaty, S., Zeitouni, O., Wu, W.: Large deviations for the two-dimensional two-component plasma (2015). arXiv:1510.01955

38. Lewin, M.: Geometric methods for nonlinear many-body quantum systems. J. Funct. Anal. 260, 35353595 (2011)

39. Lewin, M., Seiringer, R.: Strongly correlated phases in rapidly rotating Bose gases. J. Stat. Phys. 137, 10401062 (2009)

40. Lieb, E.H.: Thomas-Fermi and related theories of atoms and molecules. Rev. Mod. Phys. 53, 603$641(1981)$

41. Lieb, E.H., Loss, M.: Analysis, vol. 14 of Graduate Studies in Mathematics, 2nd edn. American Mathematical Society, Providence, RI (2001)

42. Lieb, E.H., Rougerie, N., Yngvason, J.: Rigidity of the Laughlin liquid. J. Stat. Phys. (2018). arXiv:1609.03818 (in print)

43. Lieb, E.H., Seiringer, R.: The Stability of Matter in Quantum Mechanics. Cambridge University Press, Cambridge (2010)

44. Lieb, E.H., Simon, B.: The Thomas-Fermi theory of atoms, molecules and solids. Adv. Math. 23, 22116 (1977)

45. Lundholm, D., Rougerie, N.: Emergence of fractional statistics for tracer particles in a Laughlin liquid. Phys. Rev. Lett. 116, 170401 (2016)

46. Marcus, M., Mizel, V.J.: Absolute continuity on tracks and mappings of Sobolev spaces. Arch. Ration. Mech. Anal. 45, 294-320 (1972) 
47. Martin, J., Ilani, S., Verdene, B., Smet, J., Umansky, V., Mahalu, D., Schuh, D., Abstreiter, G., Yacoby, A.: Localization of fractionally charged quasi-particles. Science 305, 980-983 (2004)

48. Papenbrock, T., Bertsch, G.F.: Rotational spectra of weakly interacting Bose-Einstein condensates. Phys. Rev. A 63, 023616 (2001)

49. Parameswaran, S.A., Roy, R., Sondhi, S.L.: Fractional quantum hall physics in topological flat bands. C. R. Phys. 14, 816-839 (2013)

50. Petrache, M., Rota-Nodari, S.: Equidistribution of jellium energy for Coulomb and Riesz interactions (2016). arXiv:1609.03849

51. Petrache, M., Serfaty, S.: Next order asymptotics and renormalized energy for Riesz interactions. J. Inst. Math. Jussieu 16, 501-569 (2014)

52. Radó, T.: Subharmonic Functions. Springer, Berlin (1937)

53. Rota Nodari, S., Serfaty, S.: Renormalized energy equidistribution and local charge balance in $2 \mathrm{~d}$ Coulomb systems. Int. Math. Res. Not. 11, 3035-3093 (2015)

54. Rougerie, N.: De Finetti theorems, mean-field limits and Bose-Einstein condensation (2014). (LMU Lecture Notes). arXiv:1506.05263

55. Rougerie, N.: Some contributions to many-body quantum mathematics (2016). (Habilitation Thesis). arXiv: 1607.03833

56. Rougerie, N., Serfaty, S.: Higher-dimensional Coulomb gases and renormalized energy functionals. Commun. Pure Appl. Math. 69, 519 (2016)

57. Rougerie, N., Serfaty, S., Yngvason, J.: Quantum Hall states of bosons in rotating anharmonic traps. Phys. Rev. A 87, 023618 (2013)

58. Rougerie, N., Serfaty, S., Yngvason, J.: Quantum Hall phases and plasma analogy in rotating trapped Bose gases. J. Stat. Phys. 154, 2-50 (2014)

59. Rougerie, N., Yngvason, J.: Incompressibility estimates for the Laughlin phase. Commun. Math. Phys. 336, 1109-1140 (2015)

60. Rougerie, N., Yngvason, J.: Incompressibility estimates for the Laughlin phase, part II. Commun. Math. Phys. 339, 263-277 (2015)

61. Rougerie, N., Yngvason, J.: The Laughlin liquid in an external potential. Lett. Math. Phys. 108, 1007-1029 (2018). https://doi.org/10.1007/s11005-017-1020-5

62. Saff, E.B., Totik, V.: Logarithmic Potentials with External Fields, vol. 316 of Grundlehren der Mathematischen Wissenschaften [Fundamental Principles of Mathematical Sciences]. Springer, Berlin (1997). (Appendix B by Thomas Bloom)

63. Saminadayar, L., Glattli, D.C., Jin, Y., Etienne, B.: Observation of the $e / 3$ fractionally charged Laughlin quasiparticle. Phys. Rev. Lett. 79, 2526-2529 (1997)

64. Sandier, E., Serfaty, S.: 1D log gases and the renormalized energy: crystallization at vanishing temperature. Probab. Theory Relat. Fields 162, 1-52 (2014)

65. Sandier, E., Serfaty, S.: 2D Coulomb gases and the renormalized energy. Ann. Probab. 43, 20262083 (2014)

66. Serfaty, S.: Coulomb Gases and Ginzburg-Landau Vortices. Zurich Lectures in Advanced Mathematics. European Mathematical Society, Zurich (2015)

67. Serfaty, S.: Microscopic description of log and Coulomb gases (2017). arXiv:1709.04089

68. Serrin, J., Varberg, D.E.: A general chain rule for derivatives and the change of variables formula for the Lebesgue integral. Am. Math. Mon. 76, 514-520 (1969)

69. Störmer, H., Tsui, D., Gossard, A.: The fractional quantum Hall effect. Rev. Mod. Phys. 71, S298S305 (1999)

70. Trugman, S., Kivelson, S.: Exact results for the fractional quantum Hall effect with general interactions. Phys. Rev. B 31, 5280 (1985)

71. Tsui, D.C., Störmer, H.L., Gossard, A.C.: Two-dimensional magnetotransport in the extreme quantum limit. Phys. Rev. Lett. 48, 1559-1562 (1982)

72. Viefers, S.: Quantum Hall physics in rotating Bose-Einstein condensates. J. Phys. C 20, 123202 (2008)

73. Zhang, Y., Sreejith, G.J., Gemelke, N.D., Jain, J.K.: Fractional angular momentum in cold atom systems. Phys. Rev. Lett. 113, 160404 (2014) 